\title{
A numerical study of crack shielding/anti-shielding in layered architectures
}

\author{
Songsong Lu ${ }^{1 *}$, Andreu Laborda ${ }^{1}$, Richard Cook$^{2}$, Yi Zhang $^{3}$, Rolandas Verbickas ${ }^{3}$, Philippa Reed ${ }^{1}$ \\ ${ }^{1}$ Mechanical Engineering Group, Faculty of Engineering and Environment, University of Southampton, UK \\ ${ }^{2}$ nCATS, Faculty of Engineering and the Environment, University of Southampton, Southampton, UK \\ ${ }^{3}$ Daido Metal Co. Ltd, European Technical Centre (UK), Winterhay Lane, IIminister, TA19 9PH, UK
}

*Songsong.Lu@soton.ac.uk; Building 5, Highfield Campus, Southampton SO17 1BJ, United Kingdom.

\begin{abstract}
Previous research has indicated the potential effectiveness of adopting micro-scale layered architectures to improve the fatigue damage resistance of coatings. However, the optimisation of these coatings is difficult due to the existence of many different factors affecting fatigue performance in such micro-scale layered architectures. In particular, the interaction of successive shielding and anti-shielding processes on crack growth resistance is therefore addressed in this paper by numerical simulation analysis. The crack tip fields in a series of layered architectures were simulated based on varying assumed constitutive materials properties to reveal the interaction of shielding/anti-shielding characteristics in complex architectures in a simple exemplar system. The evolution of crack driving force (CDF) with crack length was calculated and linked to crack growth life to investigate the effect of the ordering of shielding/anti-shielding characteristics on the crack tip field and hence on crack growth life. Layered architectures with different numbers, ordering and placing of layers, were assessed and parametric studies of 4 layered architectures were carried out. This allows analysis of the effect of these individual factors on shielding and its interactive effect with anti-shielding in multilayered architectures. Understanding the combined effect on crack growth performance and overall fatigue life controlled by crack propagation provides insight into the generic design of micro-scale layered architectures.
\end{abstract}

\section{Keywords}

Multi-layered systems, Shielding, Fatigue crack growth, Crack driving force, Finite element analysis 


\section{Introduction}

The fatigue performance of components continually needs to be improved due to more demanding service conditions in various application fields, including the automotive industry where lightweight design and durability are required [1]. Developing new/improved materials with better properties for particular performance requirements is one approach to this issue. Another approach is to develop optimised materials systems, which take advantage of our understanding of failure mechanisms based on existing materials and manufacturing techniques. One example where this application of new materials systems can be applied is the design of plain bearings, which typically adopt layered architectures [2].

Plain bearings are a key component in the internal combustion engine, which can be classified as single-layer (mono-metal), double layer (bimetal) and multi-layer (tri-metal) with layered coating architectures defined in terms of the number of mainly functional layers [3]. Apart from single-layer plain bearings, such layered architectures offer a compromise between hard and soft materials, satisfying the demanding requirements of high mechanical strength, conformability and good wear resistance properties at the same time [4]. In addition, layered structures can also contribute to the improvement of fatigue performance due to the existence of shielding/anti-shielding effects on CDF and crack deflections related to material interfaces [5].

Extensive research on fatigue crack initiation and propagation behaviour in layered architectures at different scales has been carried out. For macro-scale layered architectures, Jiang [7] carried out 4 point bending fatigue tests on plasticity-mismatched bi-material specimens composed of stainless steel and mild steel. Rozumek [8] investigated the crack growth behaviour in a steel/titanium joint under oscillatory bending. Joyce [9] assessed the fatigue properties under simplified bending conditions of a material system typically employed in plain bearings with 3 layers, including a multiphase Al-Sn-Si lining, a thin "bonding" Al interlayer, and a steel backing. These investigations confirmed that the presence of a material interface would retard the crack growth rate as the crack propagated from the side with a lower strength or stiffness material into a higher strength or stiffness material. This well-established finding is the basis of improving fatigue performance by well-designed layered architectures. For micro-scale layered architectures, Zhou [10] carried out research on the influence of Ti/TiN bilayered and multilayered films (100 nm thickness) on the axial fatigue performance of Ti46Al8Nb alloy and indicated that such multi-layered films could reduce the effective stress amplitude on the surface of the brittle substrate and increase fatigue strength by retarding fatigue crack initiation and propagation. This indicates that more micro-scale layered architectures can also be applied to improve the fatigue performance of a materials system. 
It should be noted that understanding or predicting the fatigue performance in micro-scale layered architectures is more complex than that in macro-scale layered architectures because some factors, whose effect can be neglected in macro-scale layered architectures, become more significant. For example, experimental investigations in a series of micro-scale multi-layered composites, including $\mathrm{Ni} / \mathrm{Sn}$ produced by electroplating [11] and $\mathrm{Cu} / \mathrm{Cr}$ produced by direct current (DC) magnetron sputtering [12] [13], showed significant size-dependent mechanical properties such as micro-hardness and tensile strength will increase with the decrease of layer thicknesses without changing the thickness ratio of the different layers. The effects of local microstructure variation also become much more significant in very thin layers. These size-dependent phenomena in micro-scale layered architectures have a remarkable effect on the local stress distribution and thus affects crack initiation and propagation. As a result, these factors need to be considered fully in the design of micro-scale layered architectures and the effect of each factor needs to be fully understand.

In both micro-scale and macro-scale layered architectures, the crack tip shielding (or anti-shielding) effect is an important consideration that can be used to improve the fatigue performance of layered architectures. Pioneering studies on this were first conducted by Suresh and Sugimura [6][14], [15] on crack growth behaviour in systems with elastically and plastically mismatched layers. Their finite element studies showed that there are marked changes in the effective driving force at the crack tip due to the interaction of a crack tip plastic zone with the material interface, traversing from a harder/stiffer material into a more compliant/softer material (or vice versa). Consequently, the crack tip can be shielded (crack decelerates as it approaches the interface) or anti-shielded (crack tip accelerates) from the remotely applied loads thus affecting the crack growth rate. Their work allowed investigation of the mechanism of the shielding/anti-shielding effect. Most of these findings [16]-[19] are based on the use of finite element methods (FEM) to investigate the evolution of CDF with the change in crack length in bi-materials and tri-metal materials. Little literature is found which directly links this evolution of CDF in layered architectures with the predicted crack growth life to study the shielding/anti-shielding effect and directly compare different layered architectures in terms of crack growth performance. Establishing a suitable methodology to do this will directly provide insight to materials architecture design.

In previous work, we have compared experimentally the fatigue performance of three kinds of micro-scale layered architectures, based on the number of intermetallic layer (IML) between nickel alloy and tin alloy (see Figure 1), and explored the corresponding fatigue mechanisms [20]. In this work, the 2IML coating architecture showed the best resistance to early damage in the coating. Several mechanisms were proposed, including the difference of expected shielding/anti-shielding effects in the different layered architectures on crack propagation. In this paper, we further explore 
the proposed mechanism of tuning shielding/anti-shielding effects in different layered architectures by numerical simulation analysis. The crack tip field in a series of layered architectures have been simulated based on the measured constitutive relations of simple exemplar hard and soft materials to reveal the effect of shielding/anti-shielding characteristics. The evolution of CDF with crack length was calculated (under continuum mechanics assumptions) and linked to predicted crack growth life to investigate the effect of shielding/anti-shielding characteristics of crack tip field on overall crack growth life directly. Layered architectures with a different number of layers were assessed and parametric studies of 4 layered architectures were carried out based on the effect of shielding/antishielding on crack growth performance, which will build the basis for optimising micro-scale layered architectures in the future.

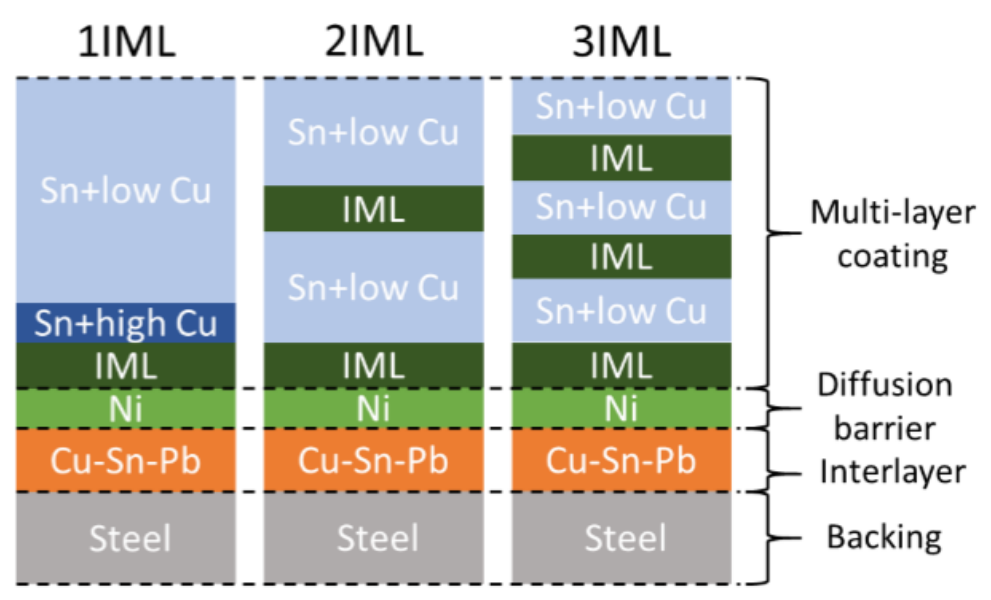

Figure 1. Cross-sectional structures of the specimens (not to scale). [20]

\section{Methodology}

\section{1. $\quad$ Simplified layered architectures}

The layered architectures shown in Figure 1 are very complex with the existence of 6 different materials and a mixture of macro and micro-scale layers. To directly model such a system with many different materials at different scales poses significant challenges in accurately identifying material properties data at different scales, meshing such fine features, incorporating size-dependent mechanical properties and allowing for the significant effect of microstructure characteristics in the modelling assumptions.

In order to simplify the numerical simulation and to specifically assess the effect of shielding/antishielding effects in different layered architectures with different combinations of soft and hard layers, 
a series of simplifications were made to represent $1 \mathrm{IML}$ and $2 \mathrm{IML}$ architectures in the numerical simulation:

a. The softer and more compliant material is allocated the known properties of the bronze material of the original interlayer in the experimental system ( $\mathrm{Cu}-\mathrm{Sn}-\mathrm{Pb})$.

b. The harder and stiffer material is allocated the known plain carbon steel properties, i.e. the backing layer in the experimental system;

c. The notional overlay thickness of the whole layered architecture is increased so that mechanical properties of the layers are not size-dependent and continuum properties can be assumed as a reasonable first order approximation;

d. All materials are considered to be homogeneous without any defects;

e. Material properties are assumed to be constant within a layer and are same for the same material in different layers.

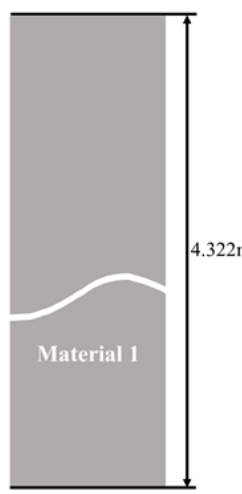

(a)

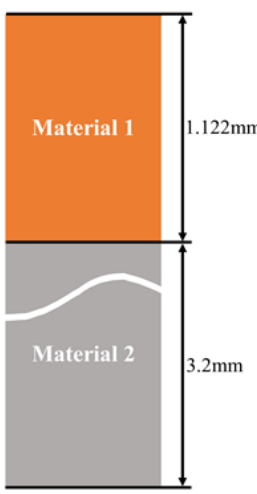

(b)

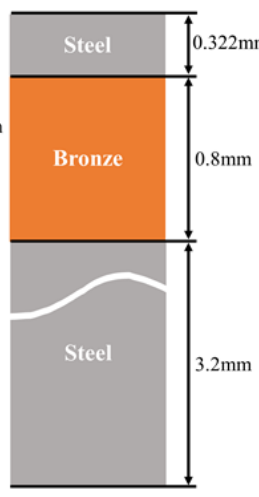

(c)

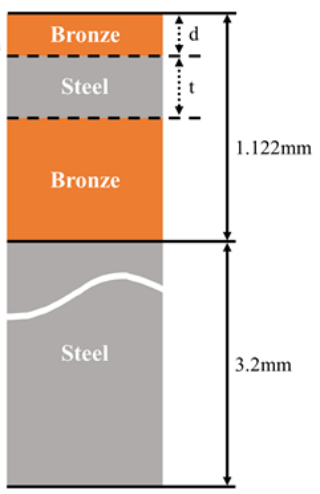

(d)

Figure 2. Schematics of cross-sectional structures of simplified layered architectures (a) Mono-metal architecture; (b) Bi-metal architecture; (c) Tri-metal architecture and (d) 4-layered architecture.

Three broad types of simplified layered architectures (see Figure 2(b)-(d)) were therefore generated. It should be noted that mono-metal architectures were considered alongside the simplified layered architectures serving as basis of comparison to assess the extent of any shielding or antishielding effects. In terms of the number of layers, these 4 broad types of simplified layered architectures are termed as mono-metal architecture, Bi-metal architecture, Tri-metal architecture and 4-layered architecture.

Each of the broad types of simplified layered architecture contains different groups of architectures (with different combinations of layer thickness and material). The detailed information of these different architectures within each broad type are as follows (see Table 1).

Table 1. List of simplified layered architectures

\begin{tabular}{l|l|l|l}
\hline \multirow{2}{*}{ Case ID } & \multirow{2}{*}{ Layered architecture type } & \multicolumn{2}{|l}{ Information for different layers (from top to bottom) } \\
\cline { 3 - 4 } & & Material & Thickness $/ \mathrm{mm}$ \\
\hline
\end{tabular}




\begin{tabular}{l|l|l|l}
\hline Case1 & Mono-metal architecture & Steel & 4.322 \\
Case2 & Mono-metal architecture & Bronze & 4.322 \\
Case3 & Bi-metal architecture & Steel+Bronze & $1.122-3.2$ \\
Case4 & Bi-metal architecture & Bronze+Steel & $1.122-3.2$ \\
Case5 & Tri-metal architecture & Steel+Bronze+Steel & $0.322-0.8-3.2$ \\
Case6 & 4-layered architecture & Bronze+Steel+Bronze+Steel & $0.322-0.2-0.6-3.2$ \\
Case7 & 4-layered architecture & Bronze+Steel+Bronze+Steel & $0.322-0.3-0.5-3.2$ \\
Case8 & 4-layered architecture & Bronze+Steel+Bronze+Steel & $0.322-0.4-0.4-3.2$ \\
Case9 & 4-layered architecture & Bronze+Steel+Bronze+Steel & $0.322-0.5-0.3-3.2$ \\
Case10 & 4-layered architecture & Bronze+Steel+Bronze+Steel & $0.422-0.3-0.4-3.2$ \\
Case11 & 4-layered architecture & Bronze+Steel+Bronze+Steel & $0.472-0.2-0.45-3.2$ \\
Case12 & 4-layered architecture & Bronze+Steel+Bronze+Steel & $0.522-0.1-0.5-3.2$ \\
Case13 & 4-layered architecture & Bronze+Steel+Bronze+Steel & $0.522-0.3-0.3-3.2$ \\
Case14 & 4-layered architecture & Bronze+Steel+Bronze+Steel & $0.622-0.3-0.2-3.2$ \\
\hline
\end{tabular}

\subsection{FE model}

The loading condition simulated in the FEM analysis are flat strips under three point bend, see Figure 3(a), these are simple fatigue testing geometries which are often used and the boundary constraints are easy to determine. Three rollers are used to apply load to the specimens and are shown in Figure $3(b)$. The distance between the two rollers contacting the top surface of the specimen is $60 \mathrm{~mm}$ while another roller is located at the center and contacts the specimen's bottom surface.

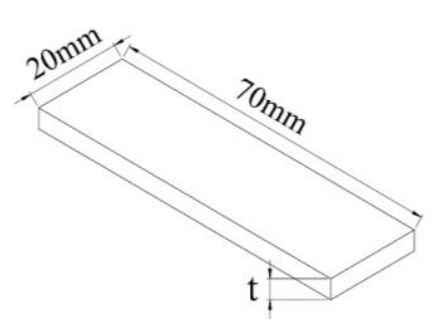

(a)

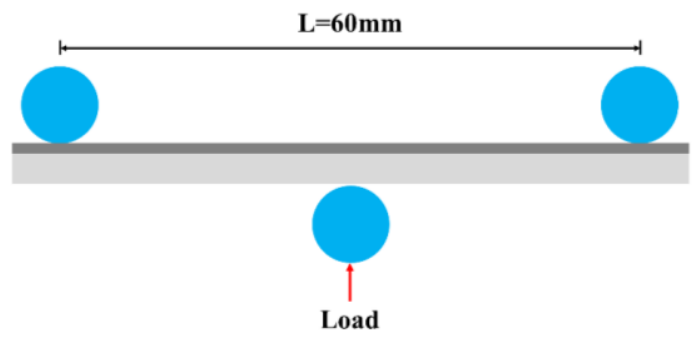

(b)

Figure 3. Configuration of (a) Flat specimens and (b) 3-point-bending tests.

Commercial software, Abaqus 6.12, was used to carry out finite element analysis and 2D FE models for simplified layered architectures under 3 point bending conditions were established. Figure 4 shows one of these assemblies which consists of half the specimen and two rollers. These two rollers are modelled as discrete rigid bodies while the flat strip is a 2D deformable plane with a vertical symmetry axis, stated as line $A C$ where Point $A$ is located at the top surface of flat strip and Point $C$ is the contact point between the flat strip and Roller-1. Point B is the crack tip marked by a white arrow in the crack surface (Line AB). It should be noted that Figure 4 is the FE model for the mono-metal architecture 
without any material interface. For other simplified layered architectures, the location of material interfaces were added onto this model.

Symmetric boundary conditions were applied to the Line BC for the symmetry of models. The line $A B$ is the straight crack along the vertical direction, whose length is stated as $a$ in this modelling work. Displacement constraints in the $\mathrm{X}$ direction are enforced on the centre of Roller-1 and all degrees of freedom for Roller-2 were fixed in order to simulate constraint on rollers from the test rig. A constant point load (50N) in the $Y$ direction was applied to the centre of Roller-1 for all crack lengthssimulated to assess the monotonic CDF under maximum loading conditions. No attempt was made to simulate cyclic loading conditions in this initial study.

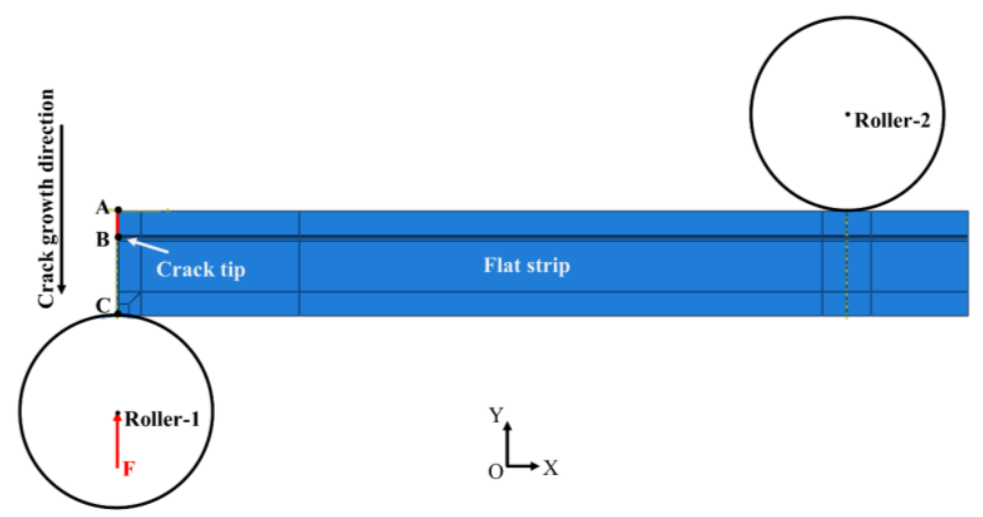

Figure 4. FE model for flat strip under 3-point-bending.

The stress-strain response of the bronze and steel were experimentally measured via tensile tests and input into the FE model as the materials constitutive models, see Figure 5, where the elastic stages are described by the measured Young's modulus and Poisson's ratio while the yield response are described by the experimentally measured data. The tensile tests were conducted on modified dogbone tensile specimens, see Figure 6, at room temperature by Instron 5569 electro-mechanical test machine with a $2 \mathrm{kN}$ load cell at a rate of $0.2 \mathrm{~mm} / \mathrm{min}$. Both strain gauges (EA-13-120LZ-120/E) and an extensometer with $10 \mathrm{~mm}$ gauge length were used to measure the strains developed during the tests. 


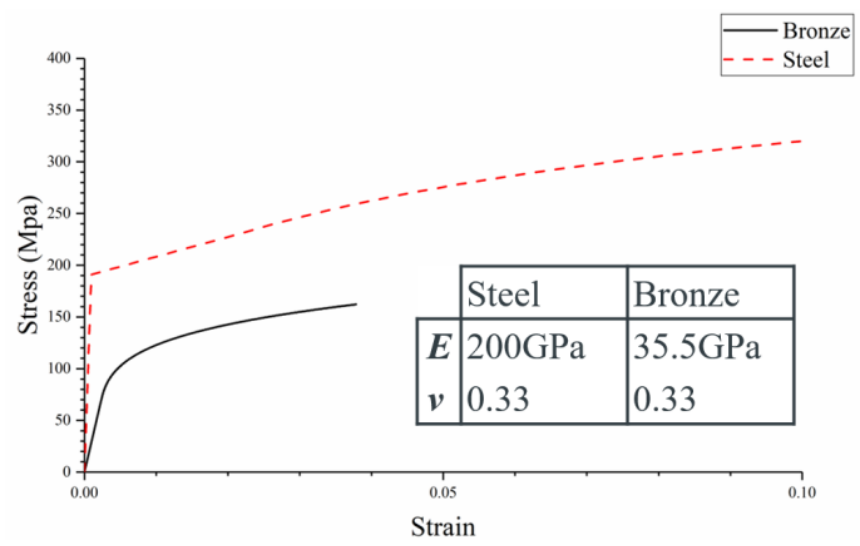

Figure 5. Stress-strain curves for bronze and steel.
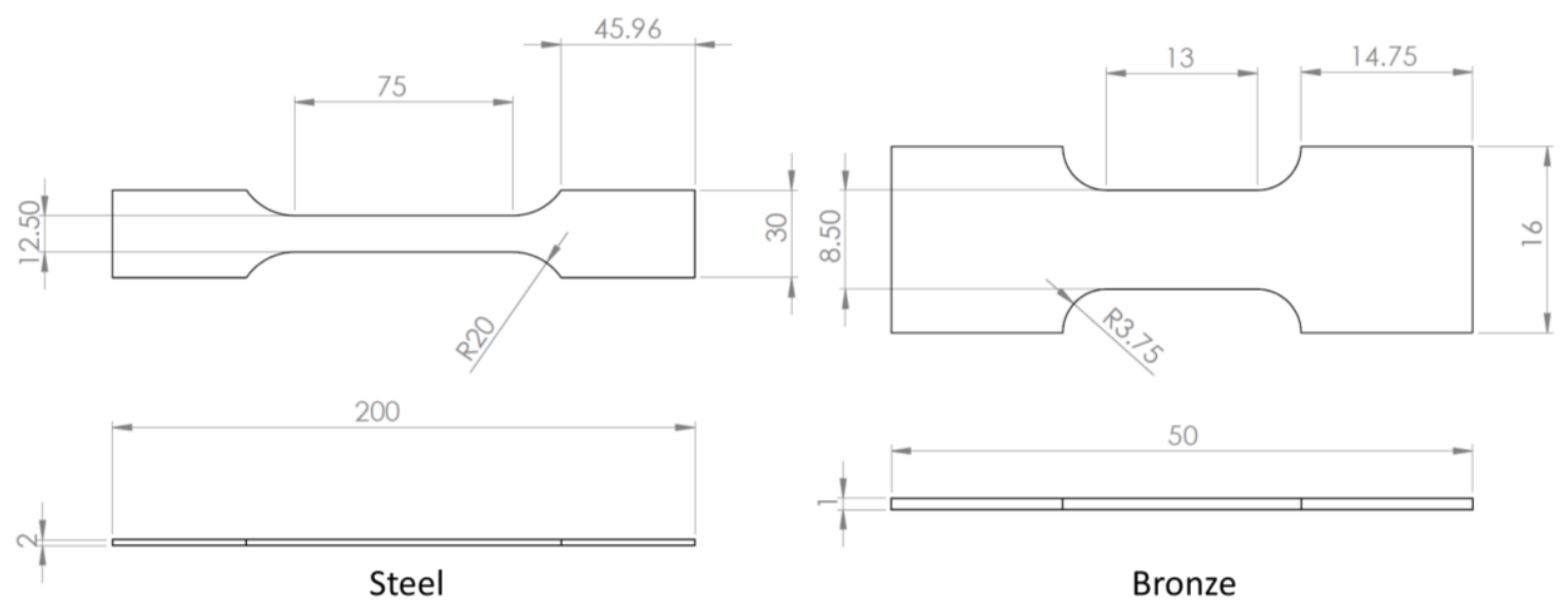

Figure 6. Steel and leaded bronze dog-bone specimens for tensile tests.

The linear plane strain element CPE4R, 4-node reduced integration bilinear plane strain quadrilateral element, was chosen to ensure efficiency of calculation because many different crack lengths are waiting to be analysed. The structure meshing technique was applied to the models due to the regular shape. Rectangle elements with sizes between $0.005 \mathrm{~mm}$ and $0.1 \mathrm{~mm}$ were generated in the flat strip model except in the area close to the crack tip. The area around the crack tip was refined and meshed in two ways (see Figure 7), based on the distance between the crack tip and the material interface. This is similar to the mesh method, which is adopted and verified by Kolednik [19]. Figure 7(a) shows the mesh for the first case where the crack tip is relatively far away from the material interface. The size of the refined mesh boundary is $0.07 \mathrm{~mm} \times 0.14 \mathrm{~mm}$. When the crack tip is near to the material interface, this refined mesh around the crack tip will be unavailable due to entering the material interface. For this case, the mesh around the crack tip is refined in another way (see Figure $7(b))$, where the material interface is located at the centre of refined area and fine elements with a similar size to that in the refined area of the first case are generated between the crack tip and the material interface. This kind of mesh around the crack tip ensures the appropriate number of elements 
are present between the crack tip and the material interface even when this distance is as low as $0.003 \mathrm{~mm}$.

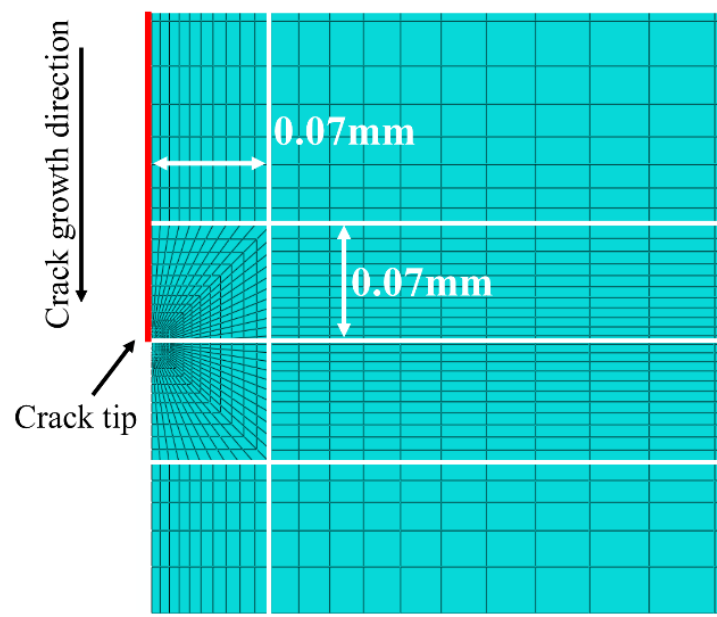

(a)

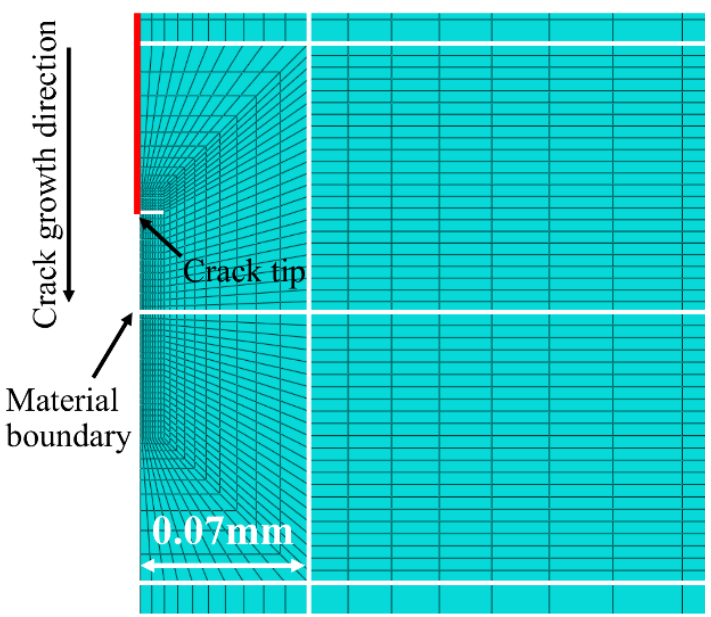

(b)

Figure 7. Mesh around crack tip when (a) the crack tip is away from the material interface and (b) near the material interface

J-integral, the CDF deemed most appropriate for heterogeneous elastic-plastic materials, was calculated in this research by the contour integral method [16]. The selected integral contour is the fifth rectangle contour around the crack tip, which never interact with the material interface.

It should be noted that comparison calculations were performed to validate the integral contour choice and the mesh method, including mesh size and refined mesh area around the crack tip, in the FE model. Firstly, the J-integral in the bimetal architecture Case4 under a series of crack lengths were calculated by selecting different integral contours, which never crossed the material interface. The maximum difference of J-integral at different crack lengths, for the integral contour around the crack tip from fifth to eighth rectangle contour, don't exceed $1.5 \%$ while their values change significantly from the second to fourth rectangle contour. This indicates that the selection of the fifth rectangle contour as the integral contour corresponds to the contour-independent characteristics of the Jintegral and verifies the effectiveness of this contour. Then, a model with similar dimension [16] was meshed by the mesh method proposed in this research. The J-integral of this model was calculated based on the same integral contour choice without changing any other parameters and compared to Joyce's original result [16]. The difference is less than $3 \%$ and indicates the validation of both the mesh method and the integral choice.

\subsection{Prediction of fatigue crack growth life}

A damage tolerance approach was adopted to predict crack growth life in the simulation by combining the predicted CDF and its relationship to crack growth rate. In this prediction, the original CDF $(J)$ is considered in terms of the equivalent stress intensity factor $(K)$, based on linear elastic 
fracture mechanics (LEFM) assumptions of small scale yielding conditions, and an assumed crack growth rate described by an appropriate Paris law.

For layered architectures, some further issues need to be considered, including the effect of discontinuous material properties on the calculation of CDF and deflection/bifurcation of cracks related to material interface. The complex crack morphology is neglected in this research, as the focus of this work is to understand the effect of shielding/anti-shielding due to discontinuous material properties on crack growth life assuming a straight crack path trajectory. Crack growth life prediction considering complex crack morphologies related to interaction with the material interface is planned as future work.

To apply this approach, three parameters therefore need to be determined for a particular layered architecture:

a. The first set of parameters (P-Type I) is related to the predicted architecture. These include the material of each layer and the location of each material interface, and specify the layered architecture.

b. The second set of parameters (P-Type II) are the mechanical properties and crack growth rate laws for all materials in the layered architecture, providing the material properties for the crack growth life prediction. Young's modulus, is needed to convert between $K$ and J-integral, and is shown in Figure 5. The Paris law constants $(C$ and $m$ ) for the steel and bronze are used in crack growth life prediction and are listed in Table 2 and plotted in Figure 8.

Table 2. List of material constants in Paris' law [21], [22]

\begin{tabular}{l|l|l|l}
\hline Material ID & Material type & $C$ & $m$ \\
\hline 1 & Steel & $3.93 \times 10^{-9}$ & 3.038 \\
2 & Bronze & $2.72 \times 10^{-10}$ & 4.18 \\
\hline
\end{tabular}

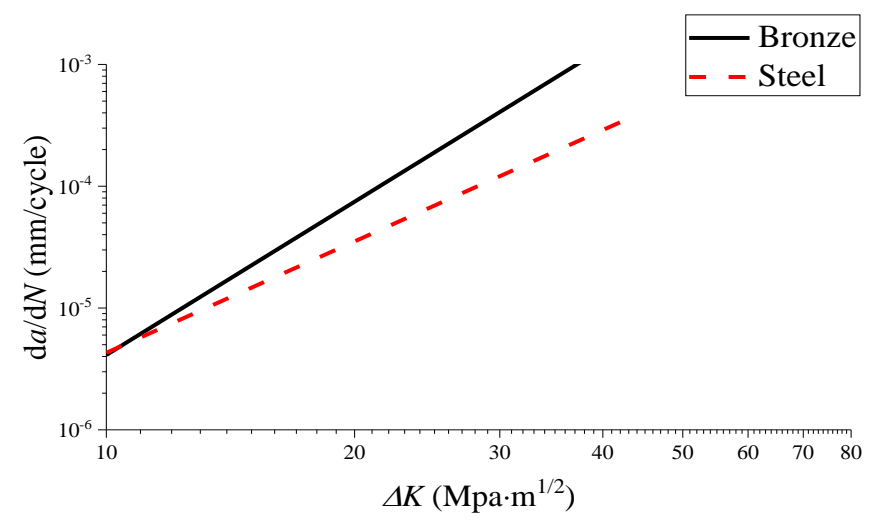

Figure 8. Crack growth life described by Paris law for steel and bronze [21], [22] 
c. The last set of parameters (P-Type III) integrate the various calculation stages and include the number of layers needed in P-Type I, the number of materials needed in P-Type II, the range of crack length, step size for the increase of crack length between calculation steps and the stress ratio. It should be noted that the range of crack length and step size are used to determine all points of crack length calculated in the crack life prediction. These parameters are listed in Table 3.

Table 3. List of P-Type III parameters

\begin{tabular}{l|l|l|l}
\hline Step size & Minimum crack length & Maximum crack length & Stress ratio \\
\hline $0.001 \mathrm{~mm}$ & $0.105 \mathrm{~mm}$ & $2 \mathrm{~mm}$ & 0.1 \\
\hline
\end{tabular}

The preliminary prediction method of crack growth life includes calculating the evolution of Jintegral with the crack length, calculating the evolution of crack growth rate with the J-integral and calculating the evolution of crack length with cycle numbers, see Figure 9.

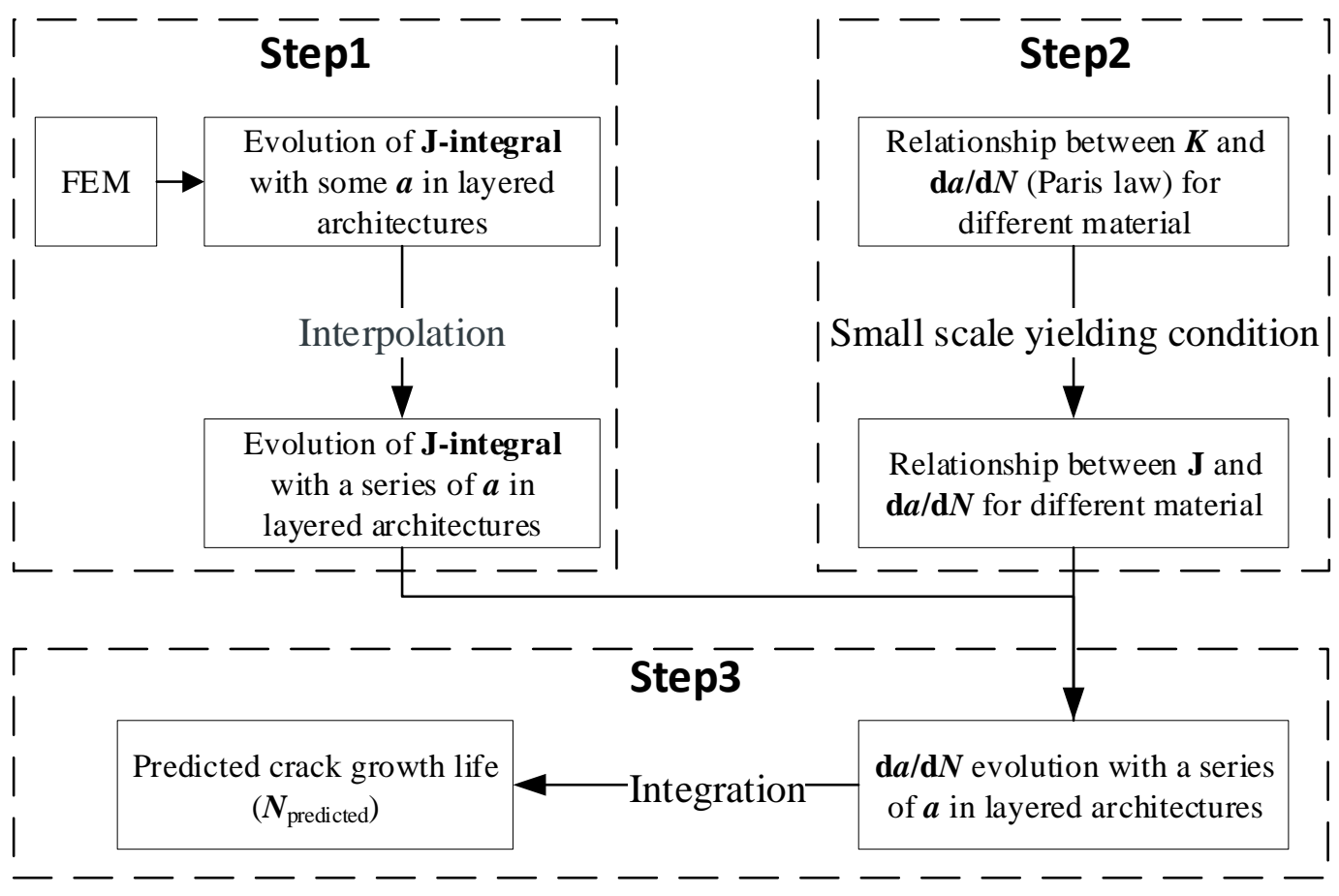

Figure 9. Flow chart of crack growth life prediction method

In the first step, FE models with different crack lengths were used to calculate the evolution of Jintegral with the crack length. The step size of crack length assessed in the FE model decreases with the distance between the crack tip and material interface. It should be noted that even the minimum step size of crack length used in the J-integral calculation is higher than the value of step size for the crack growth rate calculation shown in Table 3. The J-integral variation linked to the $0.001 \mathrm{~mm}$ step 
size in crack length adopted in the crack life predictions is therefore obtained by interpolation of the FE results.

The second step is to develop the relationship between CDF and crack growth rate. The Paris law, $\mathrm{d} a / \mathrm{d} N=C \cdot \Delta K^{m}$, relates the CDF $(\Delta K)$ to sub-critical crack growth rate $(\mathrm{d} a / \mathrm{d} N)$. The value of $\Delta K$ is calculated by $\Delta K=(1-R) \cdot K_{\max }$, where $R$ is the stress ratio and $K_{\max }$ is the maximum stress intensity factor corresponding to the maximum load applied in the FE analysis. The Paris law was linked via the relationship between J-integral and $K$ under assumed small scale yielding where $\mathrm{J}=K^{2} / E^{\prime}$, where $E^{\prime}=E$ for plane stress conditions while $E^{\prime}=E /\left(1-v^{2}\right)$ for plane strain conditions which applies here due to the geometry width and $2 \mathrm{D}$ crack assumption. As a result, the final form of the relationship is:

$$
d a / d N=C \cdot(1-R)^{m} \cdot\left(E \cdot J_{\max } /\left(1-v^{2}\right)\right)^{\frac{m}{2}}
$$

Equation 1

The third step is to predict the crack growth life at differing crack lengths. The crack growth lifetime consumed in each crack length increment for each calculation step is summed based on the relationship between crack growth rate and crack length, which is established by combining the first step and second step, as the crack growth rate is the derivative of cycle numbers with respect to crack length.

\section{Results and discussion}

\subsection{Shielding/anti-shielding crack tip fields in the bimetal architectures}

Previous research on the cracking behaviour in bimetal architectures has indicated that crack antishielding will occur when the crack grows from a hard or stiffer material into a soft or more compliant material. This anti-shielding effect on the crack tip field can also be seen (as expected) in the bimetal architectures assessed in this research. This is revealed by comparing the distribution of Von Mises stress around the crack tip in a bimetal architecture and the corresponding mono-metal architecture. It should be noted that here the corresponding mono-metal architecture is considered to be the same overall crack length in the same material as that found around the crack tip in the bimetal architecture.

Layered architectures containing different crack lengths, $1 \mathrm{~mm}$ and $1.21 \mathrm{~mm}$, are shown in Figure 10 andFigure 12, respectively. The orange lines in these pictures mark straight crack paths that start at the top surface. These two crack lengths are chosen in particular because the crack tips at these two lengths are located either side of the material interface in the bi-metal architectures showing the anti-shielding effect most clearly.

Figure 10(a) and Figure 12(a) show the distribution of Von Mises stress in the Case1 and Case2 architecture, which are mono-metal architectures made of the hard material (steel) and soft material 
(bronze) separately. These two architectures can then be compared to the Case 3 architecture, a bimetal architecture with hard top layer and soft bottom layer, for which the Von Mises stress distribution at different crack lengths are shown in Figure 10(b) and Figure 12(b). Compared with the standard butterfly shape crack tip field in the corresponding mono-metal architectures, the Von Mises stress distribution in the bimetal architecture presents clearly discontinuous characteristics and shows more serious stress concentration around the crack tip, both before and after traversing the interface.

More detailed information on the evolution of this enhanced crack tip stress concentration in this bimetal architecture are shown in Figure 11 and Figure 13. Before the crack propagates from the hard top layer into the bottom soft layer, the level of Von Mises Stress in this bimetal architecture is significantly higher than that in the corresponding mono-metal architectures along the $\mathrm{X}$ direction and the plastic zone in this bimetal architecture is significantly larger than in the corresponding monometal architecture ahead of the crack (along the $Y$ direction). After the crack penetrates into the bottom soft layer, comparing the Von Mises stress distributions in the bimetal architecture and the corresponding mono metal architecture an enhancement in stress level ahead and around the crack tip is still seen (albeit to a lesser degree than when the crack is still contained in the harder top layer). In all these comparisons, the higher stress level of the near crack tip field in this bimetal architecture compared to the corresponding mono-metal architectures, confirm the anti-shielding of the crack tip field in a bimetal architecture with a hard top layer and soft bottom layer.

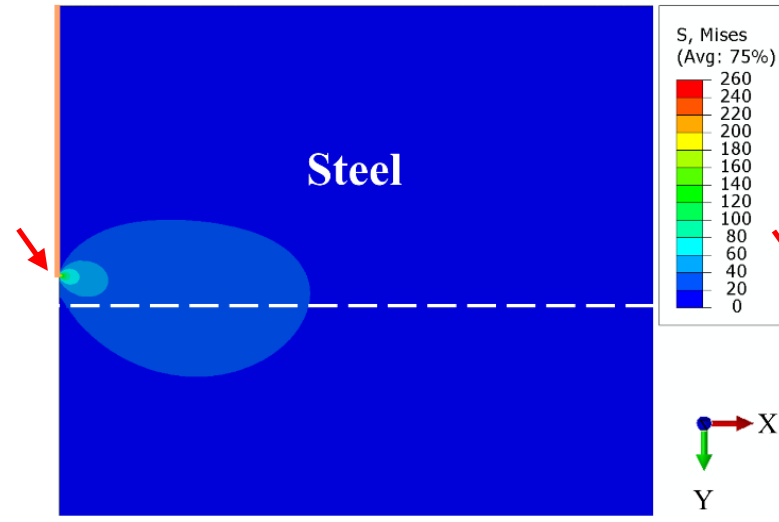

(a)

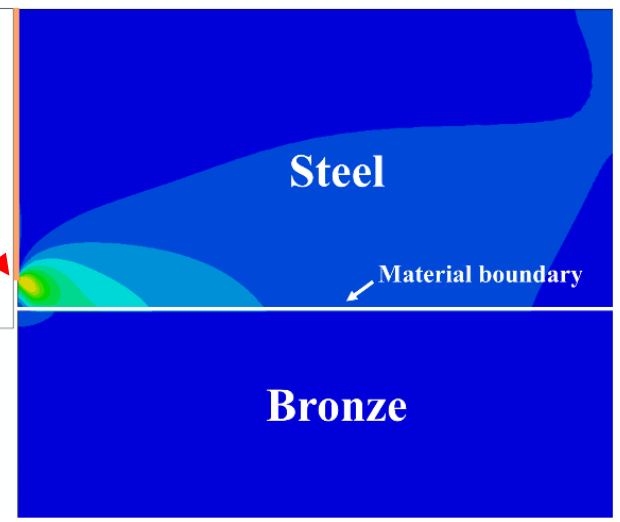

(b)

Figure 10. Von Mises stress contours with the crack length of $1 \mathrm{~mm}$ in (a) Case1 (Mono-metal architecture) and (b) Case3 (Bimetal architecture). 


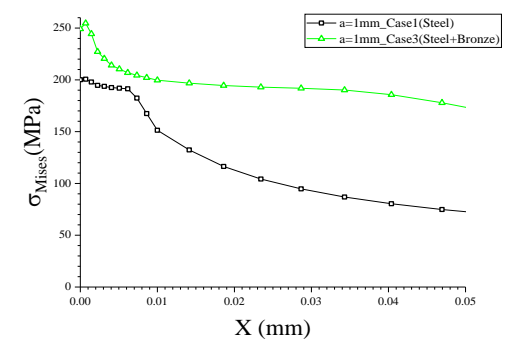

(a)

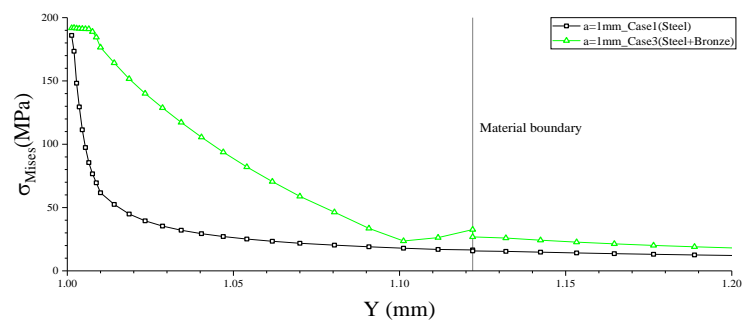

(b)

Figure 11. Von Mises stress distribution around the crack tip with the crack length of $1 \mathrm{~mm}$ along (a) $X$ direction and (b) $Y$ direction in Case1 (Mono-metal architecture) and Case3 (Bi-metal architecture).

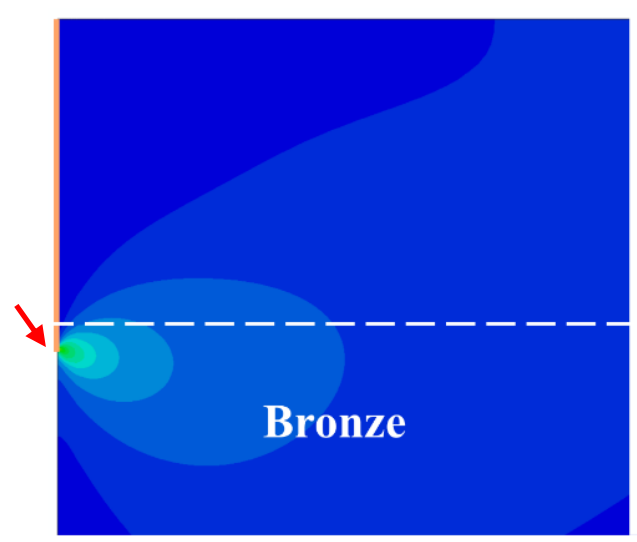

(a)

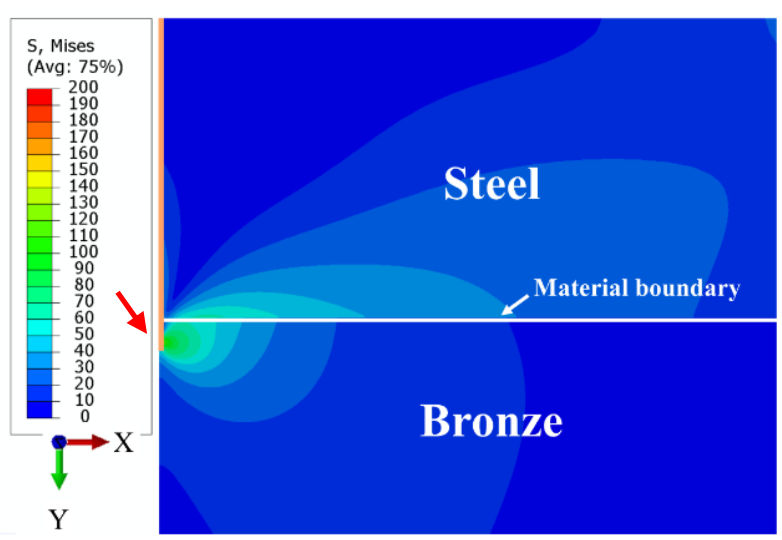

(b)

Figure 12. Von Mises stress contours with the crack length of $1.21 \mathrm{~mm}$ in (a) Case2 (Mono-metal architecture) and (b) Case3 (Bi-metal architecture).

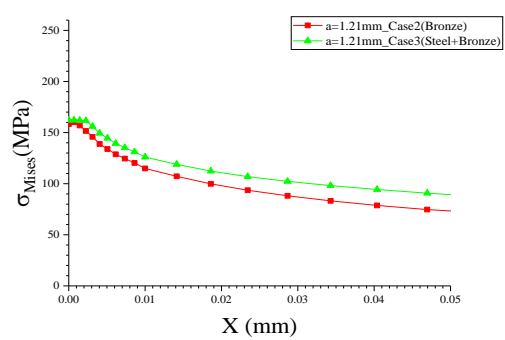

(a)

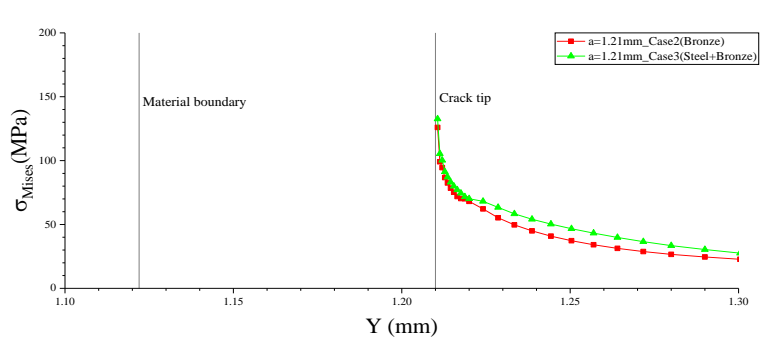

(b)

Figure 13. Von Mises stress distribution around the crack tip with the crack length of $1.21 \mathrm{~mm}$ along (a) $X$ direction and (b) $Y$ direction in Case2 (Mono-metal architecture) and Case3 (Bi-metal architecture).

Similar comparisons of the Von Mises stress distribution around the crack tip can be carried out between the shielding bimetal architecture, comprising a soft top layer and hard bottom layer, and the corresponding mono-metal architectures.

Results of these comparisons are shown in Figure 14-Figure 17 and show the opposite behavior to that in Case2. Before the crack propagates into the bottom hard layer, the level of Von Mises stress around the crack tip in this bimetal architecture is significantly lower than that in the comparable mono-metal architectures along the $X$ and $Y$ direction. It should however be noted that the stress level in this bimetal architecture does exceed that in the corresponding mono-metal architecture further ahead of the crack tip, due to the material interface and ability of the steel layer to carry a higher 
stress. After the crack penetrates into the bottom hard steel layer, the crack tip stress concentration of the layered architecture is still lower than the comparable mono-metal case (albeit a much slighter difference). These comparisons confirm the expected shielding and anti-shielding effects on crack tip stress fields in the bimetal architectures examined.

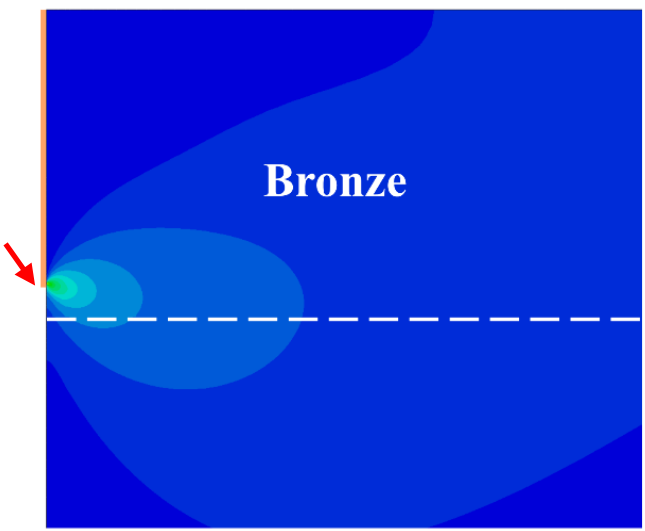

(a)

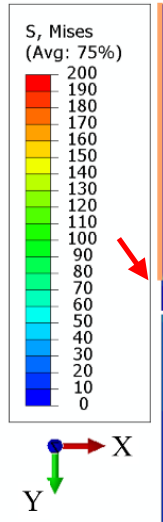

(b)

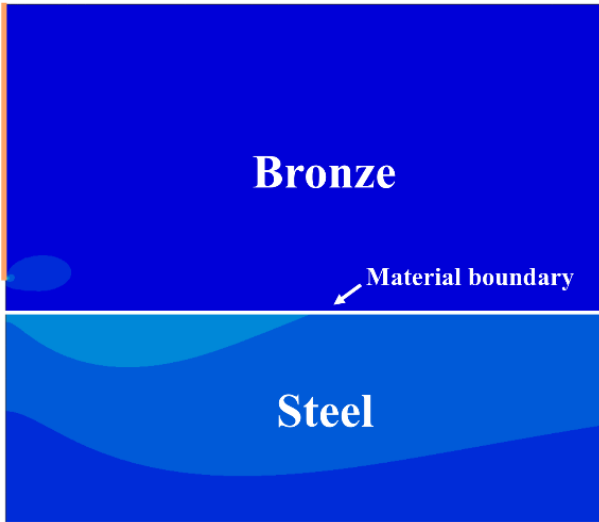

Figure 14. Von Mises stress contours with the crack length of $1 \mathrm{~mm}$ in (a) Case2 (Mono-metal architecture) and (b) Case4 (Bimetal architecture).

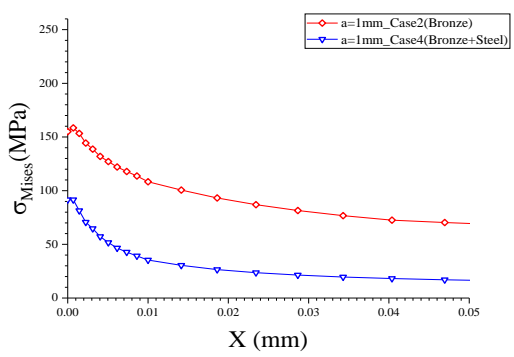

(a)

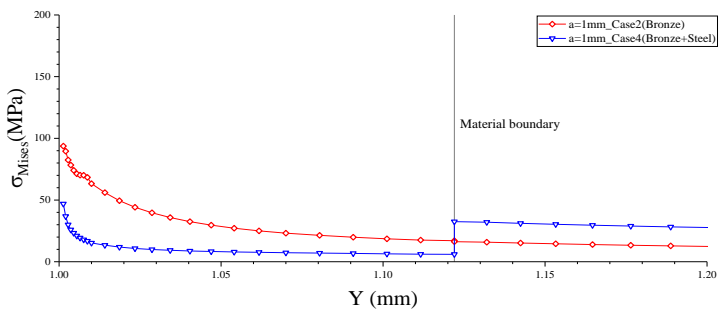

(b)

Figure 15. Von Mises stress distribution around the crack tip with the crack length of $1 \mathrm{~mm}$ along (a) $X$ direction and (b) $Y$ direction in Case2 (Mono-metal architecture) and Case4 (Bi-metal architecture).

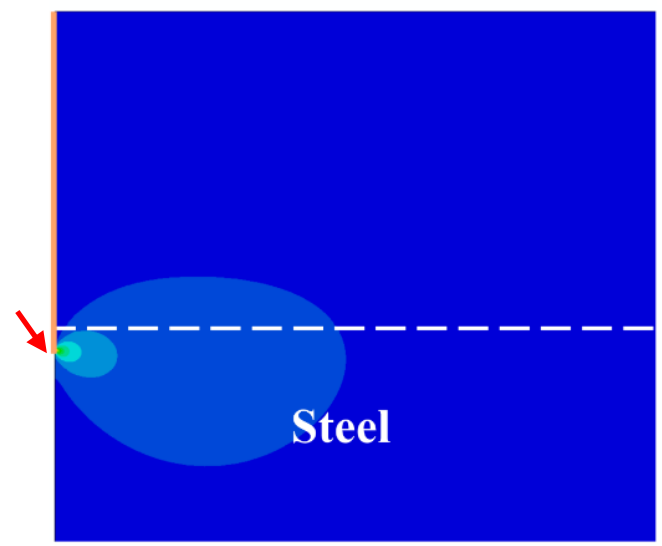

(a)

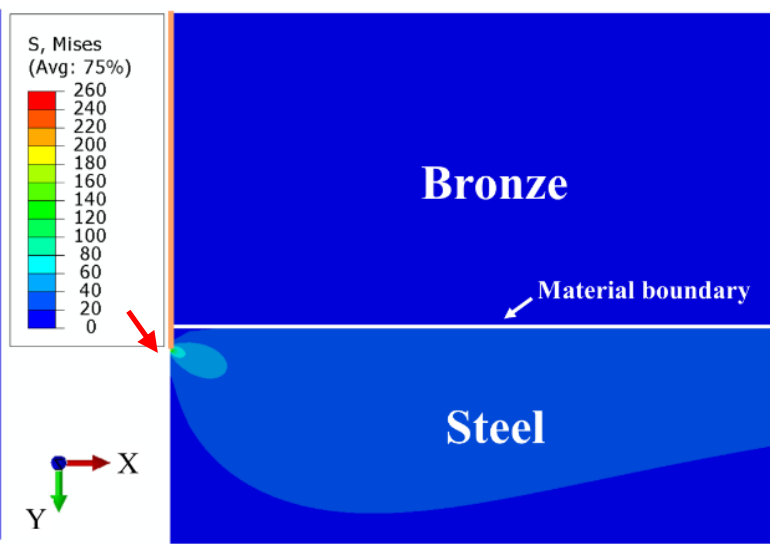

(b)

Figure 16. Von Mises stress contours with the crack length of $1.21 \mathrm{~mm}$ in (a) Case2 (Mono-metal architecture) and (b) Case4 (Bi-metal architecture). 


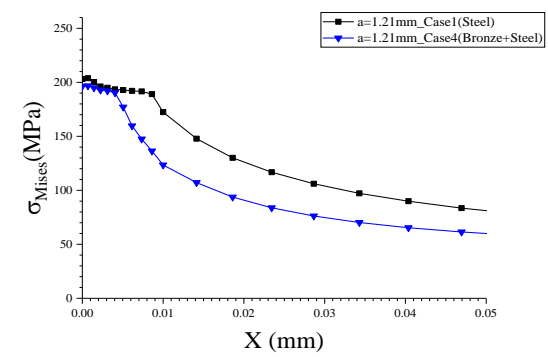

(a)

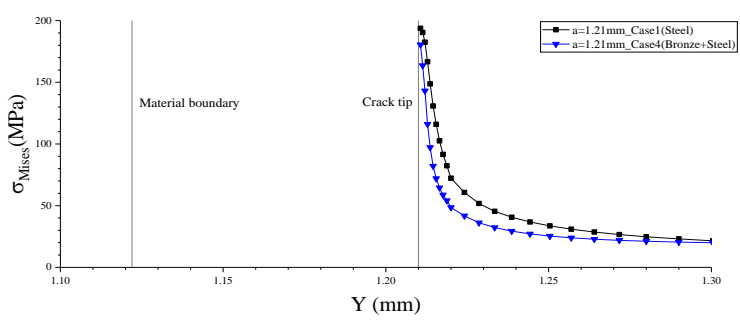

(b)

Figure 17. Von Mises stress distribution around the crack tip with the crack length of $1.21 \mathrm{~mm}$ along (a) $X$ direction and (b) $Y$ direction in Case1 (Mono-metal architecture) and Case4 (Bi-metal architecture).

The corresponding evolution of the J-integral was then calculated for these two bimetal architectures. The evolution of J-integral with crack length as it traverses the material interface is shown in Figure 18 and compared with that in the corresponding mono-metal architectures.

To understand the extent of the anti-shielding effect on the crack tip field in the Case 3 architecture, it is necessary to compare the J-integral evolution in Case3 with that in Case1 before the crack tip penetrates the material interface $(1.122 \mathrm{~mm})$ and with Case2 after the crack tip penetrates the material interface. The existence of the bottom soft layer increases the CDF in the top steel layer significantly, more than twice that seen in the single steel architecture (Case1). The impact of antishielding on the J-integral becomes more significant as the closer the crack tip comes to the material interface, increasing from 2 times $(a=0.1 \mathrm{~mm})$ to around 50 times $(a=1.115 \mathrm{~mm})$. After the crack tip enters the bottom bronze layer, the higher J-integral due to the anti-shielding effect decreases quickly, recovering to the level of that in the single bronze architecture (Case2) at a crack length around $2 \mathrm{~mm}$.

The change in shielding effect with crack length in the Case4 architectures can be seen by comparing the value of J-integral in Case4 with that in Case2 before the crack tip penetrates the material interface $(1.122 \mathrm{~mm})$ and with that in Case 1 after the crack tip penetrates the material interface. The existence of the top soft layer decreases the CDF throughout crack growth through the whole architecture. When the crack tip is in the top bronze layer, the J-integral in this bimetal architecture (Case4) is about one sixth of that in the corresponding single bronze architecture (Case2), even lower than that in the single steel architecture (Case1). The lowest J-integral in Case4 architectures is obtained just before the crack tip reaches the material interface. After the crack tip enters the bottom steel layer, the effect of shielding will start to decrease, finally recovering to the level of that in a single steel architecture at a crack length around $2 \mathrm{~mm}$. 


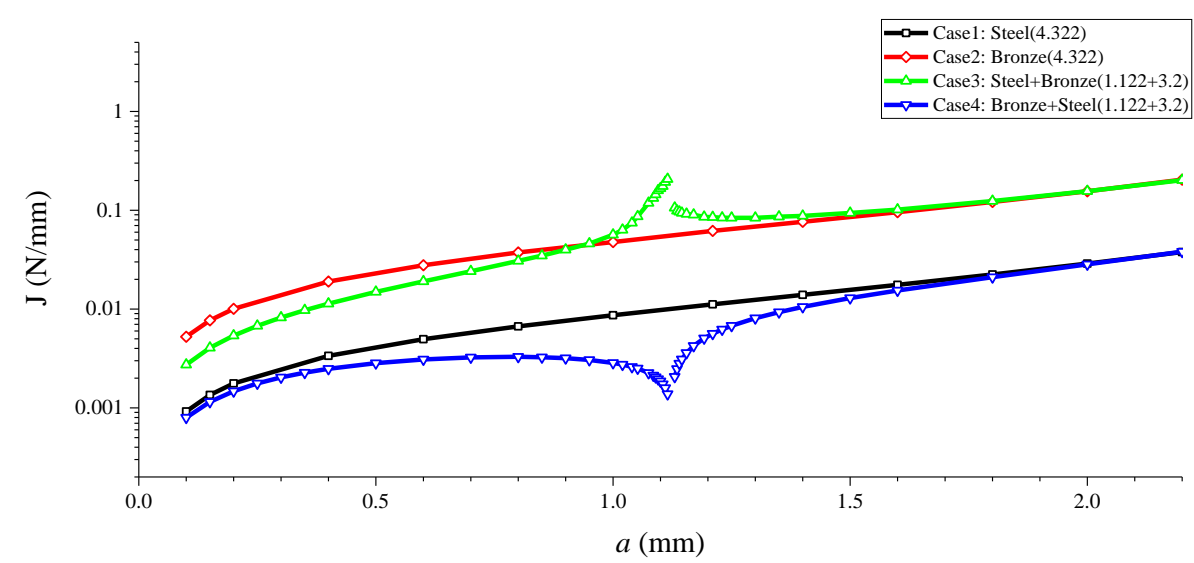

Figure 18. Evolution of J-integral with the crack length (a) in mono-metal and bimetal architectures.

\subsection{Interactive shielding and anti-shielding effects in multi-layered architectures}

In multi-layered architectures, more than one material interface exists and accordingly will affect the crack tip field. In order to investigate the effect of different material interfaces on the crack tip field, further FE analysis was carried out on models of tri-metal and 4-layered architectures, which were formed by inserting a thin hard layer into the top soft layer of the original bimetal architecture (Case14).

The crack tip stress fields are shown for a 4-layered architecture (Case14) when successive material interfaces are located just ahead of the crack tip in Figure 19 (b)-(d). The crack tip stress field in a bimetal architecture with the same crack length as that in Figure 19 (b) is also shown in Figure 19 (a) for comparison. When the crack tip is located in the bronze just before the top and bottom steel layers, the Von Mises stress contours around these two material interfaces (Figure 19 (b) and (d)) show similar characteristics to that in Figure 14 (b), indicating the expected shielding effect is operating. When the crack tip is in the first steel layer just ahead of the second bronze layer, the Von Mises stress contours (Figure 19 (c)) look similar to that seen in Figure 10(b), showing the expected anti-shielding effect.
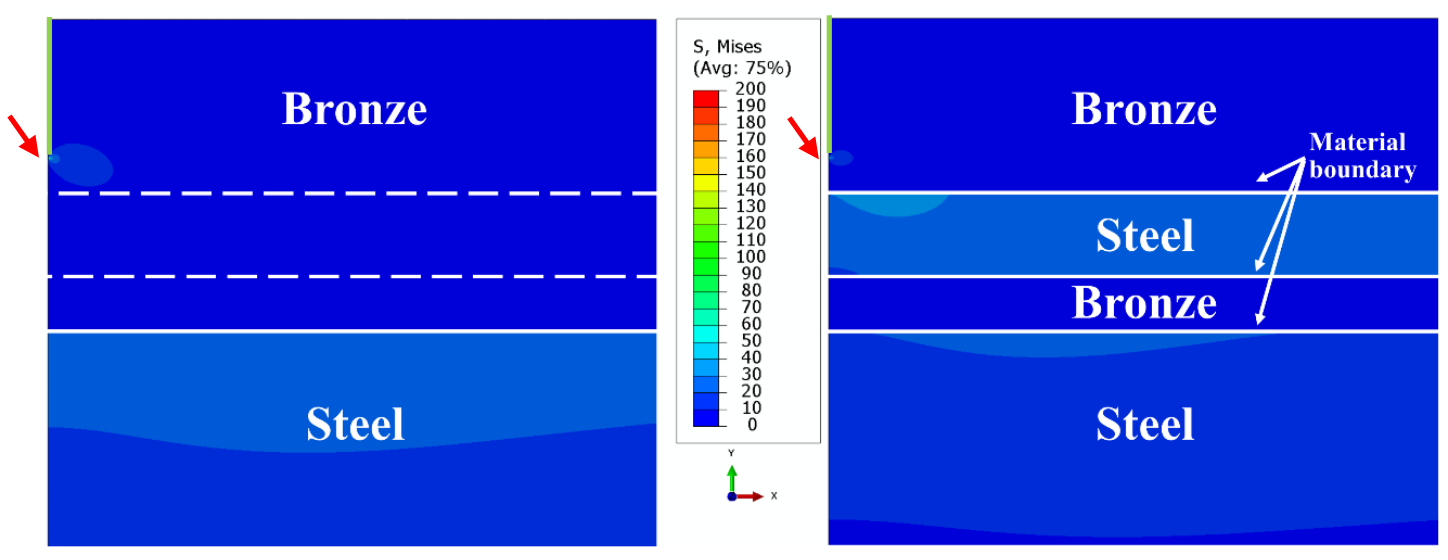
(a)

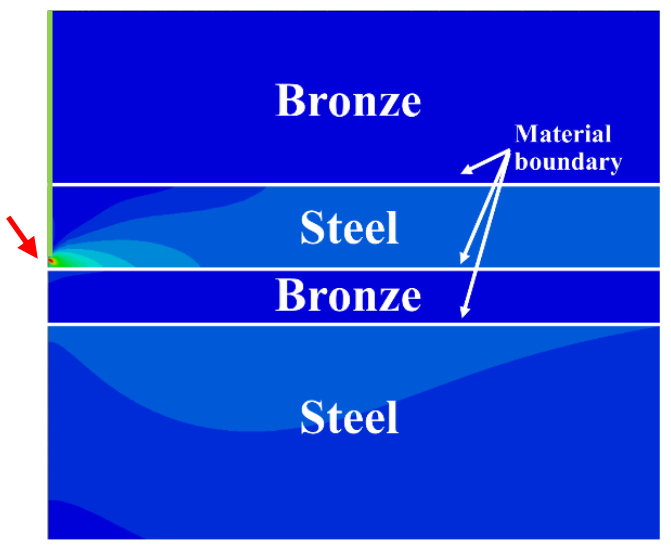

(c) (b)

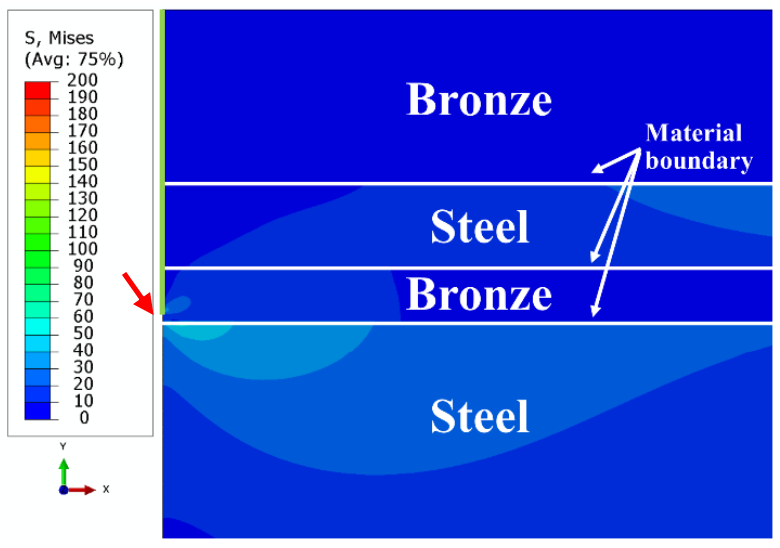

(d)

Figure 19. Von Mises stress for the crack length in (a) Case4 (Bi-metal architecture) with the crack length of $0.5 \mathrm{~mm}$; (b) Case14 (4-layered architecture) with the crack length of $0.5 \mathrm{~mm}$; (c) Case14 (4-layered architecture) with the crack length of $0.89 \mathrm{~mm}$ and (d) Case14 (4-layered architecture) with the crack length of $1.085 \mathrm{~mm}$.

With the existence of more than one material interface, a succession of shielding/anti-shielding effects will be seen on the CDF. These interactive shielding/anti-shielding effects on CDF in multilayered architectures are revealed by comparing with the J-integral found in simpler layered architectures and these can be seen in Figure 20.

Inserting thin hard layers into the top bronze layer of the bimetal architecture (Case4) will form multi-layered architectures, including the tri-metal architecture (Case5) and a 4-layered architecture (Case10). A material interface with an anti-shielding effect is one where the crack goes from a hard to a soft material. The anti-shielding effect of this material interface will also interact with shielding effect of the bottom material interface (soft to hard) on the J-integral. Part of the shielding effect of the bottom material interface will be counterbalanced by the anti-shielding effect, resulting in visible increase of J-integral in Bronze-Layer2 and small rise of J-integral in Steel-layer2 compared with that in the bimetal architecture (Case4). This material interface located at the bottom of the inserted thin hard layer is also the top material interface in tri-metal architectures, which means that its antishielding effect will dominate the level of the J-integral in the top layer. As a result, the level of Jintegral in this layer is increased significantly compared with that in the single steel architecture (Case1).

In the case of the 4-layered architecture, there is another material interface at the top surface of inserted hard layer due to the existence of the top soft layer (Bronze-Layer1). The shielding effect of this material interface dominates the level of the J-integral in the top layer and further reduces it compared with that in Case4. The shielding effect of this material interface interacts with the antishielding effect of the middle material interface, resulting in the evolution of J-integral in Steel-Layer1 which cuts across that in the single steel architecture. 


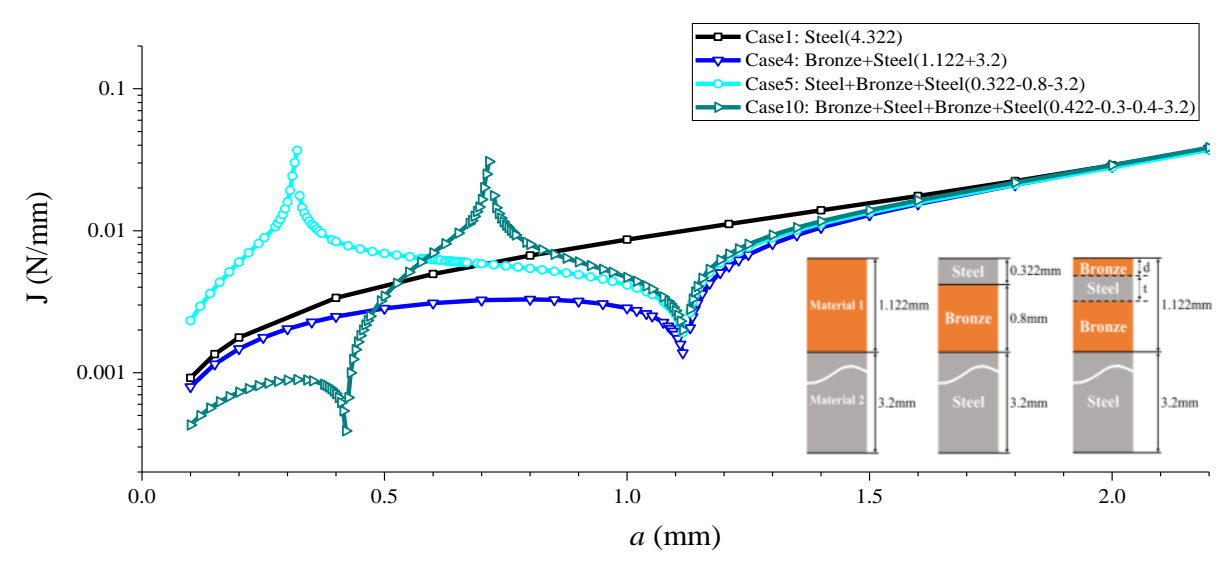

Figure 20. Evolution of J-integral with the crack length in different simplified architectures.

\subsection{Comparison of layered architectures with different numbers of layers}

Shielding/anti-shielding effects on crack tip fields in layered architectures have been shown in Sections 3.1 and 3.2 respectively. In this section, these effects on the crack tip field as the crack grows are linked to expected effects of the crack growth life directly. The crack growth life and the evolution of crack length with the number of cycles in simplified layered architectures are predicted in order to systematically compare different layered architectures in terms of optimising the shielding/antishielding effects on the evolving crack tip field. The life prediction method, stated in section 2.3, was used to carry out these crack growth lifetime predictions. The same crack growth laws for each material and applied loading conditions are adopted to allow a systematic comparison.

The ranking order of different architectures based on predicted crack growth life are shown in Figure 21. For mono-metal architectures, the steel architecture (Case1) shows better crack growth lifetime than the bronze architecture, which corresponds to the crack growth rates assigned between them based as a function of J-integral. For bimetal architectures, the bimetal architecture with a single anti-shielding event (Case3) presents the lowest predicted crack growth life (around 3000 cycles), which is less than one third of the life for the worst single architecture (Case2). A single shielding event (Case4) leads to a notable improvement in the crack growth life, more than 20 times of that in the best mono-metal architecture (Case1). The predicted crack growth life for the tri-metal architecture (Case5) is lower than that in the bimetal architecture (Case4) with shielding but is still better than that of the best mono-metal architecture (Case1). This result is due to the interaction of shielding and antishielding effects, where the anti-shielding effect occurs in the earlier stages of crack propagation and counterbalances the shielding effect due to the bottom material interface in the tri-metal architecture that has less of a contribution to overall lifetime. The highest crack growth life is presented by the 4layered architecture, Case 10, where the crack grows through a series of shielding/anti- 
shielding/shielding events which produces a lifetime more than 3 times of that in the second best layered architecture, where the bimetal architecture has a simple (single) shielding effect.

Via inspection of this ranking order, some key points can be inferred:

a. Crack growth resistance can be improved significantly by taking advantage of the shielding effect related to crack growth through a material interface growing from soft into hard materials.

b. Complex layered architectures with a top soft layer appear to have better fatigue performance. The architectures with top soft layers (Case4 and Case10) present better crack growth resistance than the corresponding Case 3 and Case5, which are bi-metal and tri-metal architectures with a top hard layer.

c. More complex layered architectures have even more potential to improve the crack growth resistance, and this is related to the degree of retardation caused by the shielding event occurring earlier in the lifetime, with a concomitant larger effect on overall lifetime. Here we see that 4layered architectures with a top soft layer are the best potential architectures in this research based on the remarkable improvements seen in overall crack growth resistance.

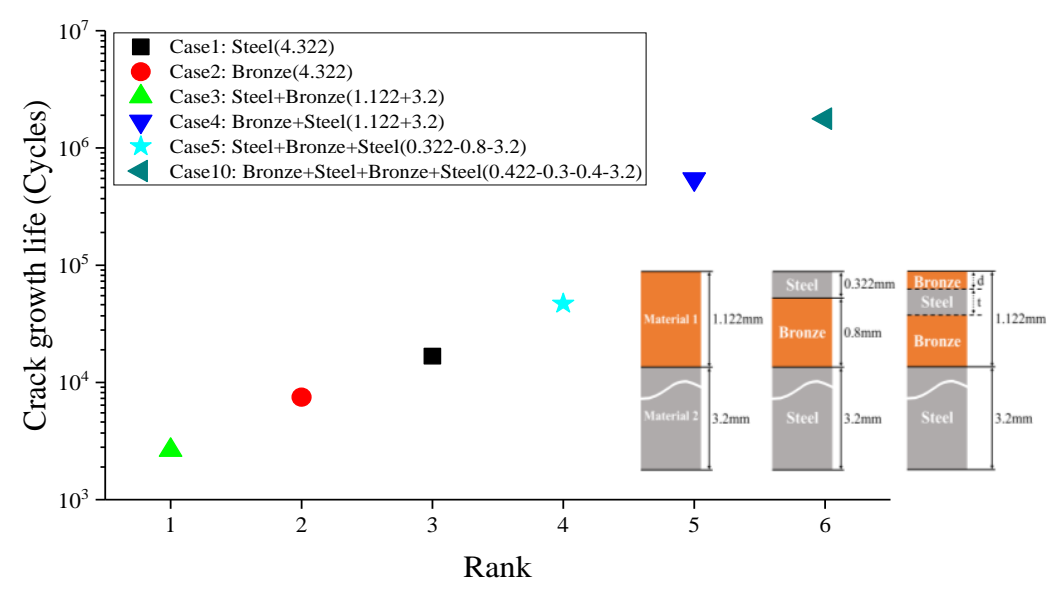

Figure 21. Rank of simplified architectures based on fatigue crack growth life prediction.

The evolution of crack length with the number of cycles in the different layered architectures are shown in Figure 22. In this graph, the crack growth life obviously depends on the structure of the layered architectures and crack growth rate changes sharply at the material interface. 


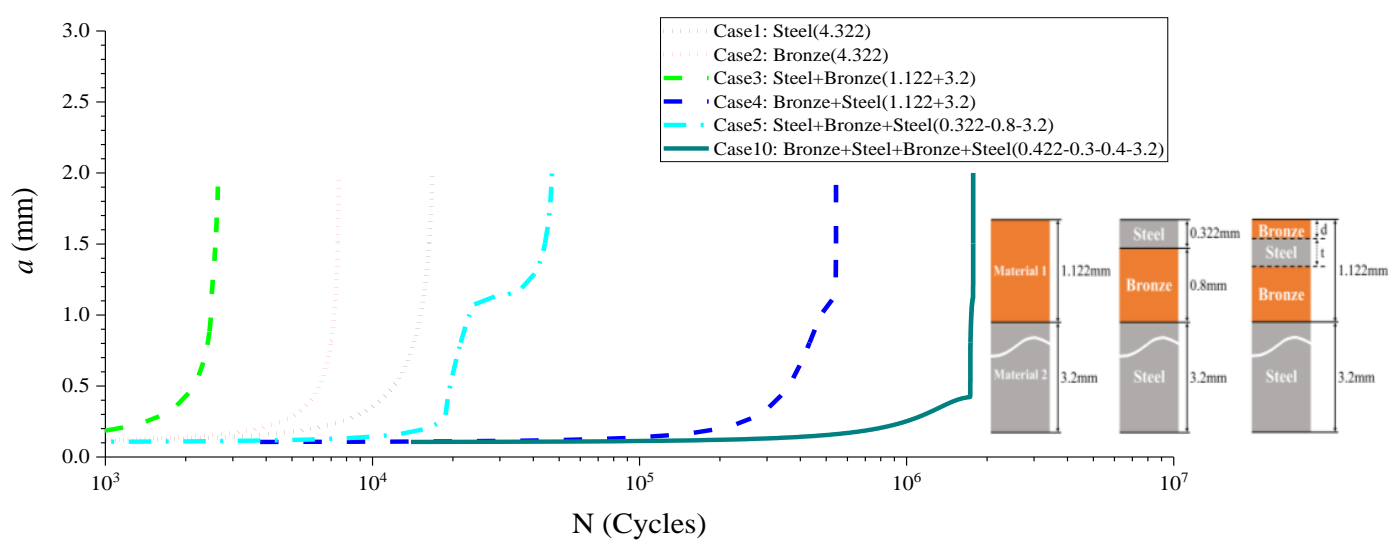

Figure 22. The evolution of crack length with cycle numbers in different simplified architectures.

In order to more fully understand the contribution of the different layers in these layered architectures to the whole crack growth life, the predicted crack growth life consumed in each layer for architectures with more than one layer are shown in Table 4. For Case10, the 4 layered architecture, most of the crack growth life (over 94\%) is consumed in the top layer. This indicates that the crack growth life consumed in the top soft layer of more complex layered architectures may be dominated most by the first shielding/anti-shielding event on crack growth life.

Table 4. The predicted crack growth life of each layer in different simplified architectures

\begin{tabular}{l|l|l|l|l|l|l|l|l|l}
\hline \multirow{2}{*}{ Case ID } & \multirow{2}{*}{$\begin{array}{l}\text { Predicted } \\
\text { life }\end{array}$} & \multicolumn{2}{|c|}{ Bronze-Layer1 } & \multicolumn{2}{c|}{ Steel-Layer1 } & \multicolumn{2}{c|}{ Bronze-Layer2 } & \multicolumn{2}{c}{ Steel-Layer2 } \\
\cline { 3 - 9 } & $\mathrm{T}$ & N Predict & $\mathrm{T}$ & N Predict & $\mathrm{T}$ & $\mathrm{N}_{\text {Predict }}$ & $\mathrm{T}$ & N $\mathrm{N}_{\text {Predict }}$ \\
\hline Case3 & $2.65 \times 10^{3}$ & - & - & 1.122 & $2.5 \times 10^{3}$ & 3.2 & $1.46 \times 10^{2}$ & - & - \\
Case4 & $5.44 \times 10^{5}$ & 1.122 & $5.41 \times 10^{5}$ & 3.2 & $2.87 \times 10^{3}$ & - & - & - & - \\
Case5 & $4.70 \times 10^{4}$ & - & - & 0.322 & $1.89 \times 10^{4}$ & 0.8 & $6.26 \times 10^{3}$ & 3.2 & $2.19 \times 10^{4}$ \\
Case10 & $1.77 \times 10^{6}$ & 0.422 & $1.71 \times 10^{6}$ & 0.3 & $1.89 \times 10^{4}$ & 0.4 & $4.04 \times 10^{4}$ & 3.2 & $2.21 \times 10^{3}$ \\
\hline
\end{tabular}

\subsection{Further parametric studies of 4-layered architectures}

The 4-layered architecture appears to be the best potential architecture in this simulation based on the crack growth resistance. In order to fully understand the crack growth performance of this architecture and to optimise multi-layered architectures, further parametric studies of the 4-layered architectures were carried out. The location and thickness of the first steel layer (Steel-layer1) were varied without changing overall thickness of the system or the thickness of the bottom steel layer (Steel-layer2).

The effect of Steel-layer1's location on the crack growth behaviour was investigated first. The location of the interface between the top bronze layer and Steel-Layer1 is represented by the thickness of the top bronze layer (d) in this research. A series of 4-layered architectures with the same 
thickness of Steel-Layer1 $(0.3 \mathrm{~mm})$ positioned at different depths, $d$, are analysed: Case7, Case10, Case13 and Case14 architectures.

The effect of Steel-Layer1's location on the evolution of J-integral with crack length is shown in Figure 23. In the top bronze layer, the J-integral decreases with the decrease of $d$. Thus having the location of Steel-layer1 very near the top surface will increase the proportion of crack growth life consumed in a very thin top soft layer in a 4-layered architecture. It can be seen that the transition in J-integral value growing through the first steel layer from the maximum shielding effect to the maximum anti-shielding effect is similar for these architectures and the effect of different architectures on the crack growth performance in this layer appears minimal.

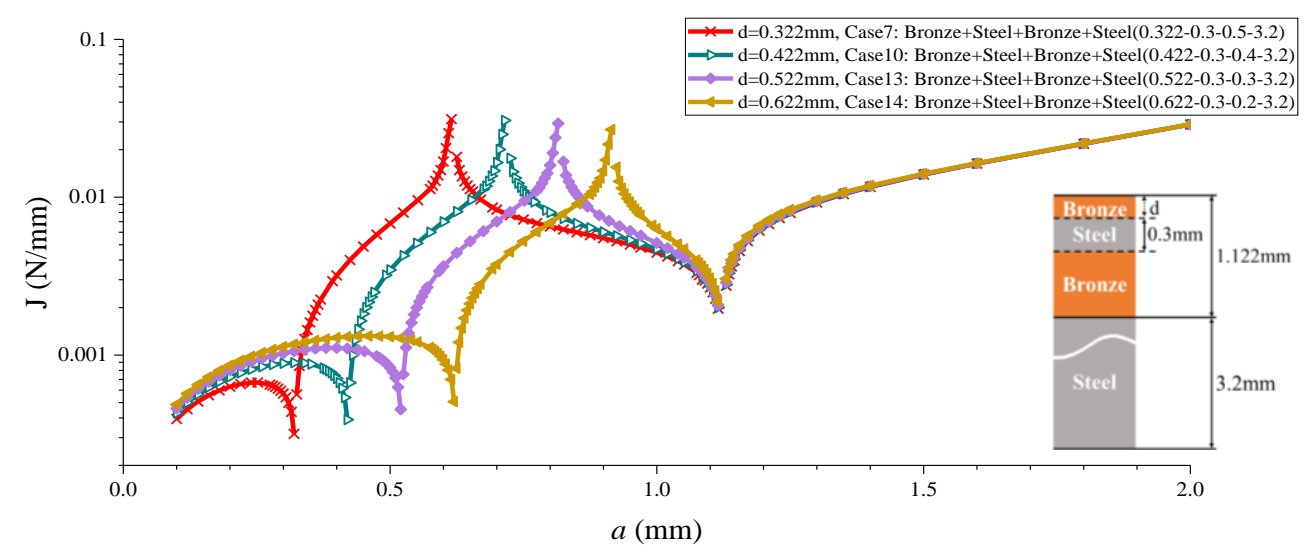

Figure 23. Evolution of J-integral with the crack length in 4-layered architectures under the effect of Steel-Layer1's location.

The evolution of crack length with the number of cycles in these 4-layered architectures with varying $d$ are also shown in Figure 24 and the corresponding crack growth life consumed in each layer are shown in Table 5. This indicates a 33\% improvement in crack growth life via changing Steel-Layer1's location, for $d$-values between $0.622 \mathrm{~mm}$ to $0.322 \mathrm{~mm}$. It should be noted that all layers contribute to this improvement. The crack growth life consumed in Bronze-Layer1, which occupies about $96 \%$ of whole propagation life, increases by $31 \%$. Subsequent growth in Steel-Layer1 and then Bronze-Layer2, shows significantly increase of crack growth life, more than doubling, but this has little effect on overall lifetime. Growth into Steel-Layer2 takes up no more than $0.2 \%$ crack growth life and crack growth lifetime in this layer only increases by $8 \%$, showing that the critical area to optimise is the very top layers of the architecture in this simulation. 


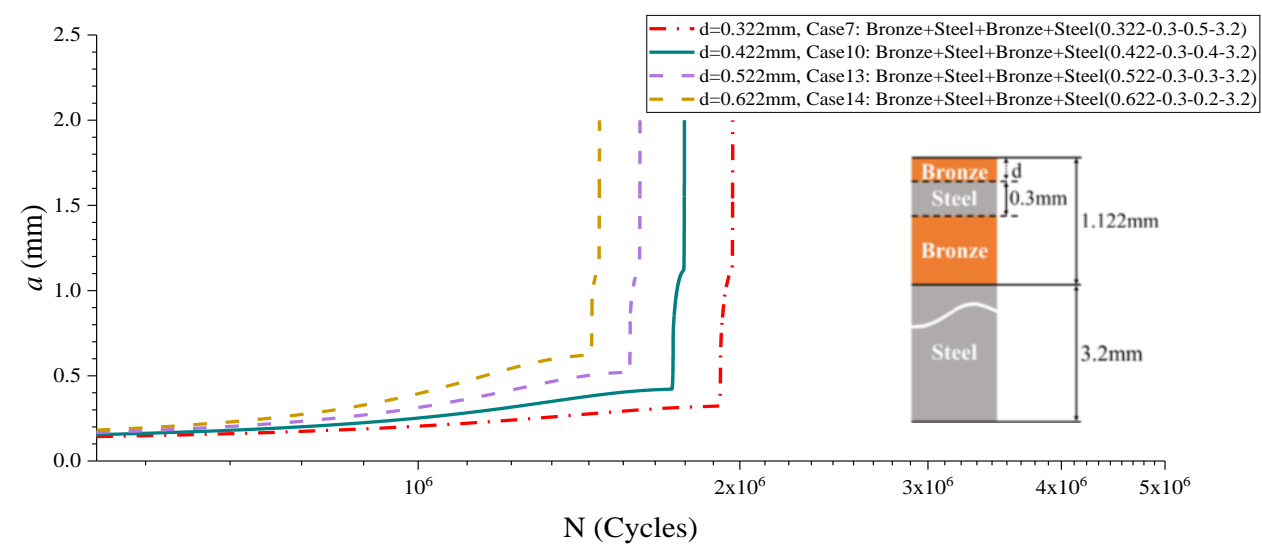

Figure 24. Crack length evolution with the numbers of cycles under the effect of Steel-Layer1's location in 4-layer architectures.

Table 5. The predicted crack growth life of each layer in 4-layered architectures with different Steel-Layer1 locations

\begin{tabular}{c|c|c|c|c|c|c|c|c|c}
\hline \multirow{2}{*}{ Case ID } & \multirow{2}{*}{$\begin{array}{c}\text { Predicted } \\
\text { life }\end{array}$} & \multicolumn{2}{|c|}{ Bronze-Layer1 } & \multicolumn{2}{c|}{ Steel-Layer1 } & \multicolumn{2}{c|}{ Bronze-Layer2 } & \multicolumn{2}{c}{ Steel-Layer2 } \\
\cline { 5 - 10 } & & $\mathrm{T}$ & $\mathrm{N}_{\text {Predict }}$ & $\mathrm{T}$ & $\mathrm{N}$ Predict & $\mathrm{T}$ & $\mathrm{N}_{\text {Predict }}$ & $\mathrm{T}$ & $\mathrm{N}_{\text {Predict }}$ \\
\hline Case7 & $1.97 \times 10^{6}$ & 0.322 & $1.89 \times 10^{6}$ & 0.3 & $2.69 \times 10^{4}$ & 0.5 & $4.87 \times 10^{4}$ & 3.2 & $2.24 \times 10^{3}$ \\
Case10 & $1.77 \times 10^{6}$ & 0.422 & $1.71 \times 10^{6}$ & 0.3 & $1.89 \times 10^{4}$ & 0.4 & $4.04 \times 10^{4}$ & 3.2 & $2.21 \times 10^{3}$ \\
Case13 & $1.61 \times 10^{6}$ & 0.522 & $1.56 \times 10^{6}$ & 0.3 & $1.47 \times 10^{4}$ & 0.3 & $3.15 \times 10^{4}$ & 3.2 & $2.16 \times 10^{3}$ \\
Case14 & $1.48 \times 10^{6}$ & 0.622 & $1.44 \times 10^{6}$ & 0.3 & $1.24 \times 10^{4}$ & 0.2 & $2.16 \times 10^{4}$ & 3.2 & $2.08 \times 10^{3}$ \\
\hline
\end{tabular}

Next, the effect of Steel-layer1's location and thickness on the crack growth performance are investigated based on analysis of more 4-layered architectures, including Case9, Case10, Case11 and Case12. For these architectures, the location of the first steel layer's mid-plane is kept constant, at $0.572 \mathrm{~mm}$ below the surface, and the Steel-layer1's thickness $(\mathrm{t})$ changes.

The effect of changing Steel-Layer1's thickness on the evolution of J-integral with crack length is shown in Figure 25. With the increase of Steel-Layer1's thickness, the maximum shielding effect related to the first interface being closer to the top surface is seen and the anti-shielding effect occurs deeper into the coating. 


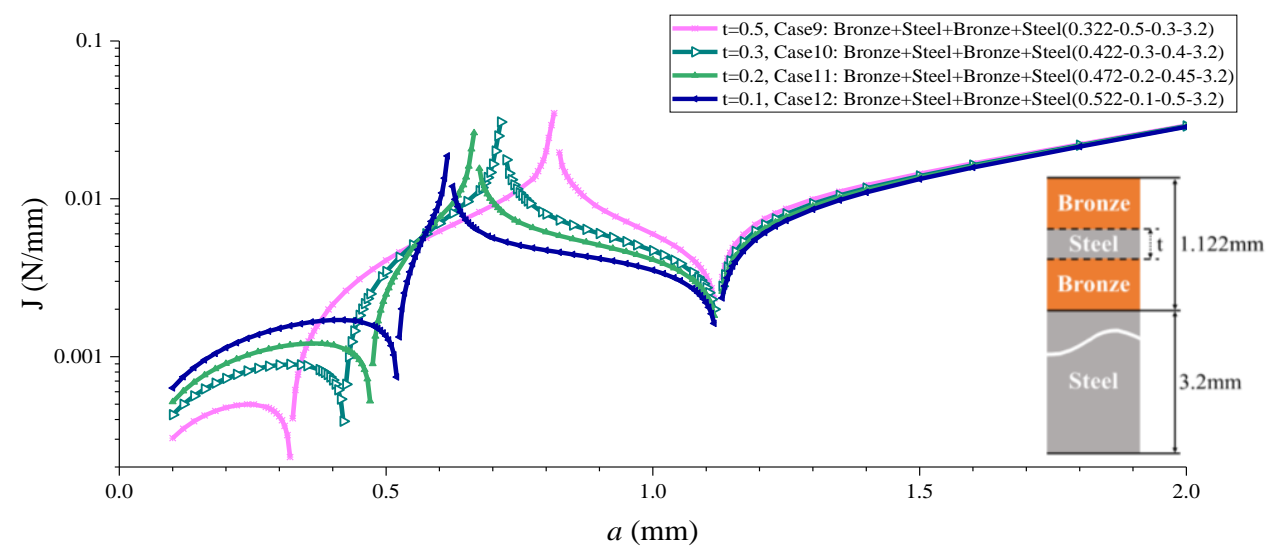

Figure 25. Evolution of J-integral with the crack length in 4-layered architectures under the effect of Steel-Layer1's thickness

The evolution of crack length with the number of cycles in 4-layered architectures with different Steel-Layer1 thicknesses are shown in Figure 26 and the corresponding crack growth life consumed in each layer are shown in Table 6. This indicates a remarkable improvement, a tripling of crack growth life in the 4-layered architecture via changing the Steel-Layer1 thickness, with $t$ increasing from $0.1 \mathrm{~mm}$ to $0.5 \mathrm{~mm}$. It should be noted that the top two layers make the most significant contributions to this improvement. The crack growth life consumed in Bronze-Layer1, occupies about $89 \%$ of the propagation life with the $0.1 \mathrm{~mm}$ Steel-layer 1 and increases to about $98 \%$ with the $0.5 \mathrm{~mm}$ Steel-layer1. Growth in Steel-Layer1 increases even more significantly, by more than 10 times the previous value. In both Bronze-Layer2 and Steel-Layer2, crack growth life consumed in these layers decreases with the increase of Steel-layer1's thickness. However, they occupy no more than $10 \%$ of total life, making the overall effect of their lifetime decrease unimportant.

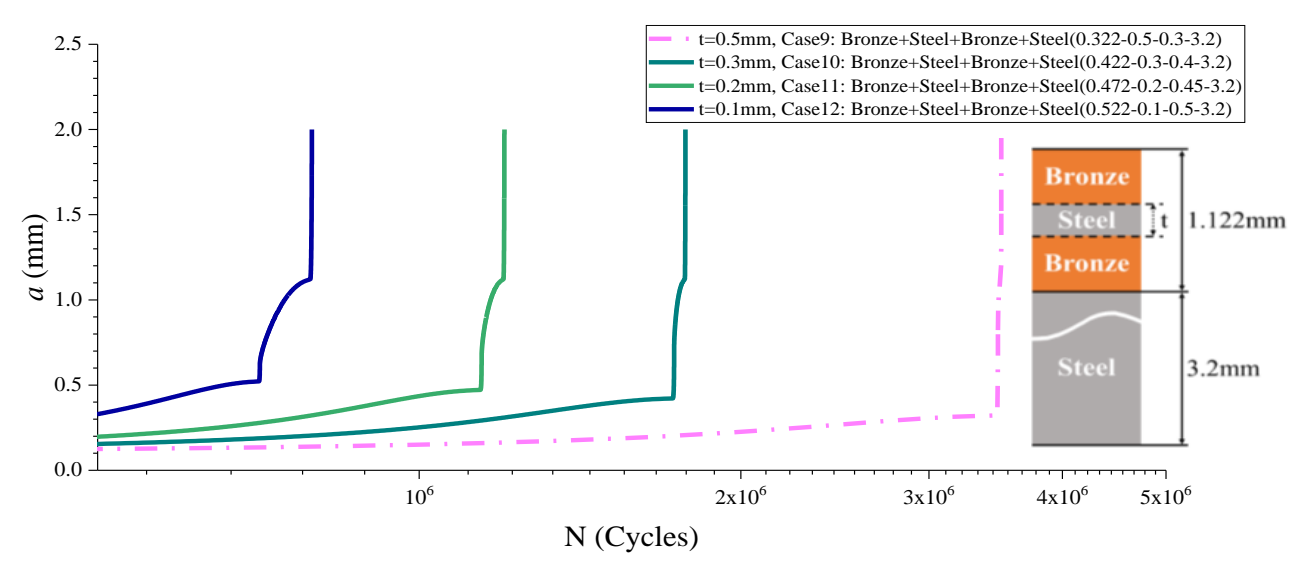

Figure 26. Crack length evolution with cycle numbers under the effect of Steel-Layer1's thickness in 4-layer architectures.

Table 6. The predicted crack growth life of each layer in 4-layered architectures with different Steel-Layer1's thickness

\begin{tabular}{c|c|c|c|c|c|c|c|c|c}
\hline \multirow{2}{*}{ Case ID } & \multirow{2}{*}{$\begin{array}{c}\text { Predicted } \\
\text { life }\end{array}$} & \multicolumn{2}{|c|}{ Bronze-Layer1 } & \multicolumn{2}{|c|}{ Steel-Layer1 } & \multicolumn{2}{c|}{ Bronze-Layer2 } & \multicolumn{2}{|c}{ Steel-Layer2 } \\
\cline { 3 - 10 } & & $\mathrm{T}$ & NPredict & $\mathrm{T}$ & NPredict & $\mathrm{T}$ & NPredict & $\mathrm{T}$ & NPredict \\
\hline
\end{tabular}




\begin{tabular}{c|c|c|c|c|c|c|c|c|c} 
Case9 & $3.51 \times 10^{6}$ & 0.322 & $3.43 \times 10^{6}$ & 0.5 & $5.21 \times 10^{4}$ & 0.3 & $2.34 \times 10^{4}$ & 3.2 & $1.97 \times 10^{3}$ \\
Case10 & $1.77 \times 10^{6}$ & 0.422 & $1.71 \times 10^{6}$ & 0.3 & $1.89 \times 10^{4}$ & 0.4 & $4.04 \times 10^{4}$ & 3.2 & $2.21 \times 10^{3}$ \\
Case11 & $1.20 \times 10^{6}$ & 0.472 & $1.13 \times 10^{6}$ & 0.2 & $1.04 \times 10^{4}$ & 0.45 & $5.52 \times 10^{4}$ & 3.2 & $2.36 \times 10^{3}$ \\
Case12 & $7.93 \times 10^{5}$ & 0.522 & $7.04 \times 10^{5}$ & 0.1 & $4.78 \times 10^{3}$ & 0.5 & $8.16 \times 10^{4}$ & 3.2 & $2.56 \times 10^{3}$ \\
\hline
\end{tabular}

The thickness of the top soft layer may however be an important factor, related to wear resistance, or the ability to entrap wear particles/debris, in the design of a coating. Thus maintaining the thickness of the top soft layer may be necessary, so to investigate this option further 4 layered architectures with the same thickness of Bronze-Layer1 $(0.322 \mathrm{~mm})$ are compared, but now varying $t$ (Steel-Layer 1 thickness) these were designated Case6, Case7, Case8 and Case9.

The interactive effect of Steel-Layer1's thickness and location on the evolution of the J-integral with crack length is shown in Figure 27. Increasing Steel-Layer1's thickness without changing the thickness of Bronze-Layer1 also increases the crack growth life as shown in Figure 28.

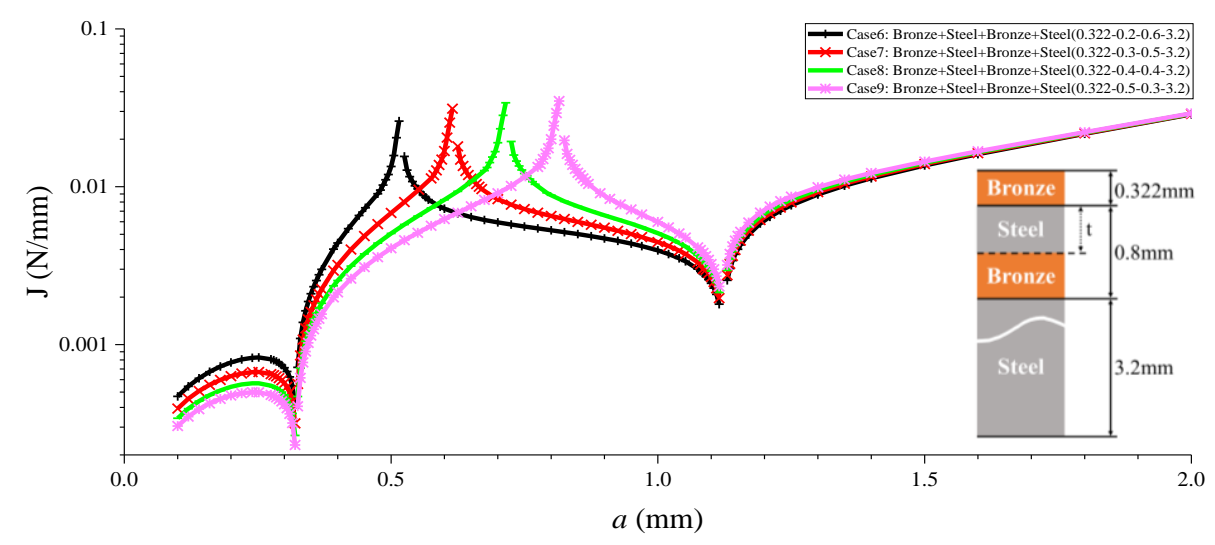

Figure 27. Evolution of J-integral with the crack length in 4-layered architectures under the interactive effect of Steel-Layer1's location and thickness.

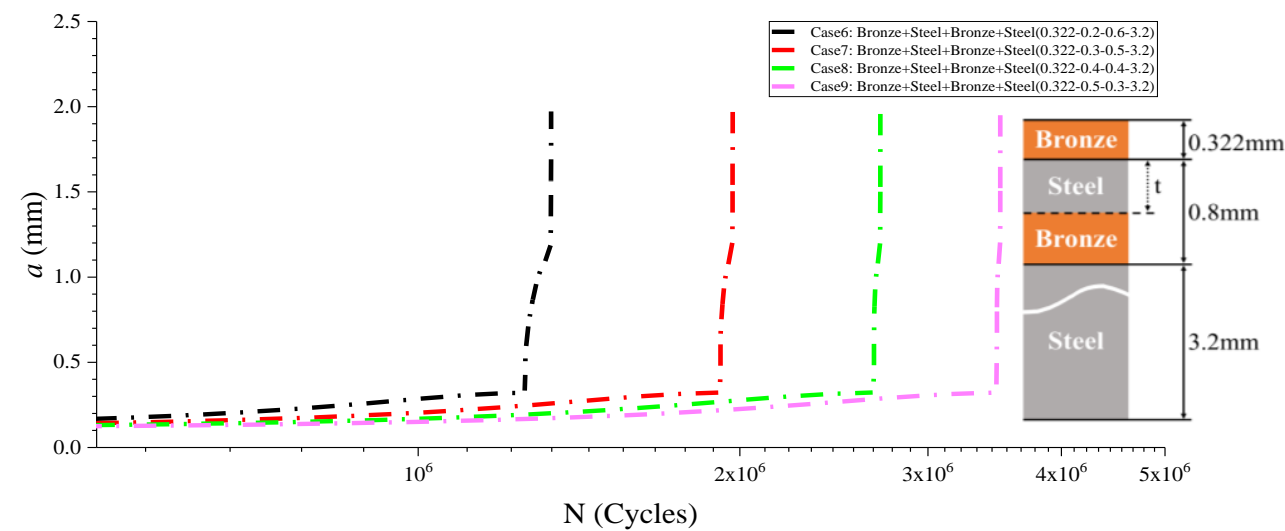

Figure 28. Crack length evolution with cycle numbers under the interactive effect of Steel-Layer1's thickness and location in 4-layer architectures when the thickness of Bronze-Layer1 keep the constant $(0.322 \mathrm{~mm})$. 
The interactive effect of Steel-Layer1's location and thickness are shown in Figure 29 based on the predicted crack growth life corresponding to the same maximum crack length $(2 \mathrm{~mm})$ for all 4-layer architectures simulated in this research. The different of Steel-Layer1's location is distinguished by d, where the value of $d$ is the thickness of the top bronze layer (Bronze-Layer1). The different thicknesses of Steel-Layer1 is distinguished by t. The positive effect of a thicker Steel-Layer1 and smaller distance between Steel-Layer1's location and the top surface are shown clearly.

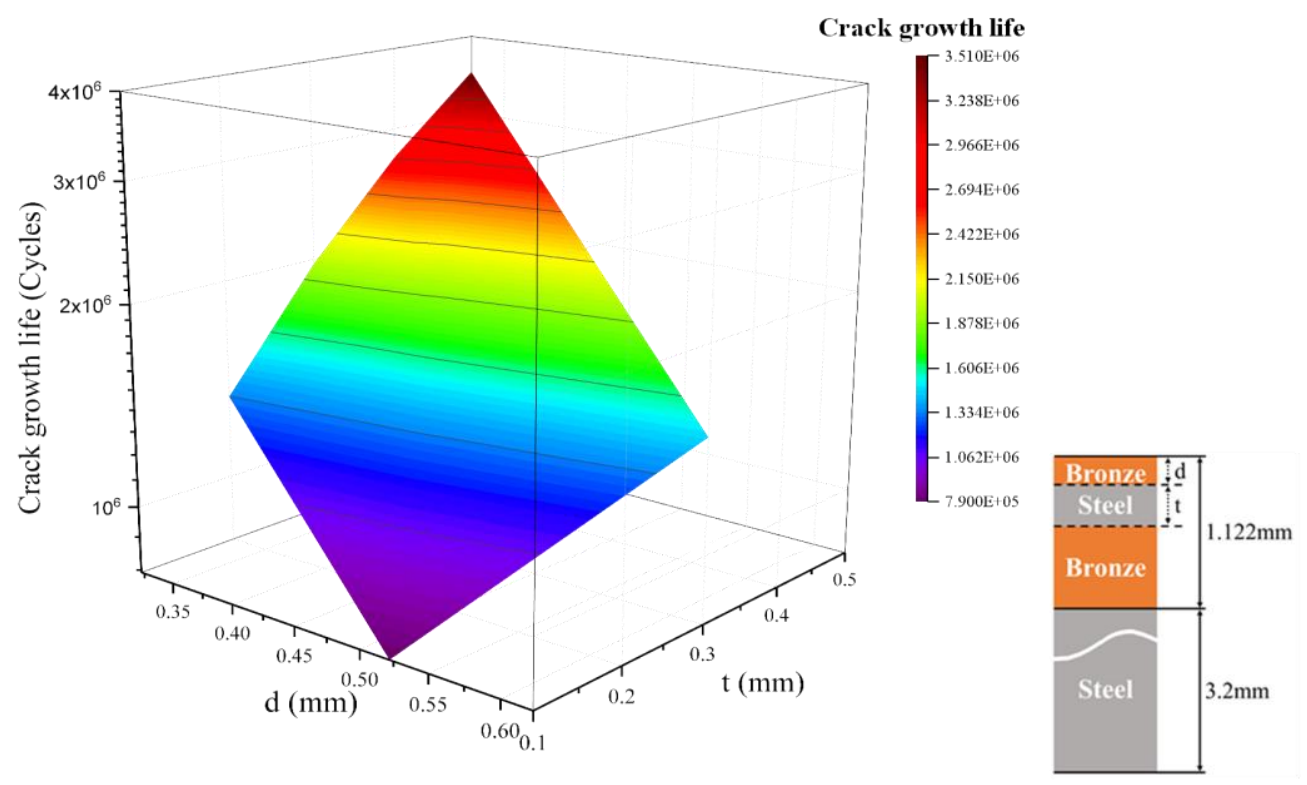

Figure 29. Results of fatigue crack growth life prediction for 4-layer architectures with different parameters of Steel-Layer1.

\section{Conclusions}

In this study, crack shielding/anti-shielding in bimetal architectures is reviewed and the interaction of shielding and anti-shielding sequences in multi-layered architectures are evaluated. A numerical simulation methodology has been established by simulating the evolution of CDF with crack length through the layers and linking it to predicted crack growth life based on a simple Paris approach. Layered architectures with different numbers of layers were compared and parametric studies of 4layered architectures were carried out based on this method. It should be noted that the present methodology doesn't assess the effect of crack path deflection or model the early stages of crack propagation. This methodology aims to particularly clarify the expected magnitude of the effect of shielding/anti-shielding characteristics on a simplified crack tip field and to assess how this related to predicted crack growth performance. The following conclusions about macro-scale layered architectures can be drawn from the numerical simulation and analysis of this paper, but their applicability in micro-scale layered architectures will need further research: 
1) In multi-layered architectures, interactions of shielding and anti-shielding effects related to transitions across different material interfaces can have a significant effect on the level of CDF.

2) Complex layered architectures with a top soft layer will provide better fatigue performance because the top material interface will experience a significant shielding effect that will provide effective crack propagation resistance by decreasing the level of CDF in the early crack growth stage that controls the majority of fatigue life.

3) For multi-layered architectures with a top soft layer, the crack growth performance in the top soft layer dominates the multi-layered architectures' overall crack growth performance. Thus the comparison of multi-layered architectures based on crack growth performance may be carried out by focussing analysis on the top soft layer.

4) For multi-layered architectures with a top soft layer, increasing the thickness of the first hard layer and decreasing the distance between it and the top surface will provide higher crack propagation resistance. The effect of first hard layer's thickness is more significant than the effect of its location.

\section{Acknowledgements}

This work was supported by the Engineering and Physical Sciences Research Council (EPSRC, EP/N509747/1) and Daido Metal Co. Ltd.

The accompanying data presented in this paper can be found via the doi: https://doi.org/10.5258/SOTON/D0743.

\section{References}

[1] Bajpai, R. and Chandrasekhar, U., Innovative Design and Development Practices in Aerospace and Automotive Engineering, 2017, Springer.

[2] Zhang, Y., Tudela, I., Pal, M. and Kerr, I., High strength tin-based overlay for medium and high speed diesel engine bearing tribological applications. Tribology International, 2016, 93, pp.687-695.

[3] Kopreliovich, D., Bearing in internal combustion engines [online], www.substech.com [Accessed 30 July 2018].

[4] Kopreliovich, D., Requirements to engine bearing materials [online], www.substech.com [Accessed 30 July 2018].

[5] Burke-Veliz, A., Syngellakis, S. and Reed, P.A.S., Fatigue crack shielding and deflection in plain bearings under large-scale yielding. Engineering Failure Analysis, 2010, 17(3), pp.648-657.

[6] Suresh, S., Sugimura, Y. and Tschegg, E.K., The growth of a fatigue crack approaching a perpendicularly-oriented, bimaterial interface. Scripta metallurgica et materialia, 1992, 27(9), pp.1189-1194.

[7] Jiang, F., Deng, Z.L., Zhao, K. and Sun, J., Fatigue crack propagation normal to a plasticity mismatched bimaterial interface. Materials Science and Engineering: A, 2003, 356(1-2), pp.258-266.

[8] Rozumek, D. and Bański, R., Crack growth rate under cyclic bending in the explosively welded steel/titanium bimetals. Materials \& Design, 2012, 38, pp.139-146. 
[9] Joyce, M.R., Syngellakis, S. and Reed, P.A.S., Fatigue crack initiation and early growth in a multiphase Al alloy included in a multilayer material system. Materials science and technology, 2004, 20(1), pp.47-56.

[10] Zhou, Y., Rao, G.B., Wang, J.Q., Zhang, B., Yu, Z.M., Ke, W. and Han, E.H., Influence of Ti/TiN bilayered and multilayered films on the axial fatigue performance of Ti46Al8Nb alloy. Thin Solid Films, 2011, 519(7), pp.2207-2212.

[11] Wang, W. and Singh, R.N., Influence of the microstructure on the mechanical properties of Ni/Sn multilayered composites. Materials Science and Engineering: A, 1999, 271(1-2), pp.306-314.

[12] Zhang, J.Y., Zhang, X., Liu, G., Zhang, G.J. and Sun, J., Scaling of the ductility with yield strength in nanostructured Cu/Cr multilayer films. Scripta Materialia, 2010, 63(1), pp.101-104.

[13] Niu, J.J., Zhang, J.Y., Liu, G., Zhang, P., Lei, S.Y., Zhang, G.J. and Sun, J., Size-dependent deformation mechanisms and strain-rate sensitivity in nanostructured $\mathrm{Cu} / \mathrm{X}(\mathrm{X}=\mathrm{Cr}, \mathrm{Zr}$ ) multilayer films. Acta Materialia, 2012, 60(9), pp.3677-3689.

[14] Sugimura, Y., Lim, P.G., Shih, C.F. and Suresh, S., 1995. Fracture normal to a bimaterial interface: effects of plasticity on crack-tip shielding and amplification. Acta Metallurgica et Materialia, 43(3), pp.1157-1169.

[15] Sugimura, Y., Grondin, L. and Suresh, S., 1995. Fatigue crack growth at arbitrary angles to bimaterial interfaces. Scripta Metallurgica et Materialia, 33(12).

[16] Joyce, M.R., Reed, P.A.S. and Syngellakis, S., Numerical modelling of crack shielding and deflection in a multi-layered material system. Materials Science and Engineering: A, 2003, 342(1-2), pp.11-22.

[17] Burke-Veliz, A., Reed, P.A. and Syngellakis, S., A numerical study of crack shielding and deflection under extensive plasticity. Engineering Fracture Mechanics, 2009, 76(9), pp.1345-1356.

[18] Burke-Veliz, A., Syngellakis, S. and Reed, P.A.S., Fatigue crack shielding and deflection in plain bearings under large-scale yielding. Engineering Failure Analysis, 2010, 17(3), pp.648-657.

[19] Kolednik, O., Predan, J., Shan, G.X., Simha, N.K. and Fischer, F.D., On the fracture behavior of inhomogeneous materials-A case study for elastically inhomogeneous bimaterials. International Journal of Solids and Structures, 2005, 42(2), pp.605-620.

[20] Laborda, A., Robinson, A., Wang, S., Zhang, Y. and Reed, P., Fatigue assessment of multilayer coatings using lock-in thermography. Materials \& Design, 2018, 141, pp.361-373.

[21] Wang, Y., Gao, Z. and Zhang, F., Study on fatigue crack growth of 316L steel at different temperatures. Pressure Vessel, 2004, 21(6), pp.6-7. (In Chinese)

[22] Zhang, Y., Li, Y., Liang, J., et al., Fatigue crack propagation characteristics of ZCuAl8Mn14Fe3Ni2 copper alloy for propeller. Development and Application of Materials, 2010, 25(5), pp.1-3. (In Chinese) 